\title{
Markov Partitions for Dispersed Billiards ${ }^{\star}$
}

\author{
L. A. Bunimovich and Ya. G. Sinai \\ L. D. Landau Institute for Theoretical Physics, Academy of Sciences, SU-117334 Moscow, USSR
}

\begin{abstract}
Markov Partitions for some classes of billiards in two-dimensional domains on $\mathbb{R}^{2}$ or two-dimensional torus are constructed. Using these partitions we represent the microcanonical distribution of the corresponding dynamical system in the form of a limit Gibbs state and investigate the character of its approximations by finite Markov chains.
\end{abstract}

\section{Dispersed Billiards and Formulation of Main Results}

Let $Q$ be a two-dimensional open bounded connected domain on $\mathbb{R}^{2}$ or the twodimensional torus with Euclidean metric. We suppose that the boundary $\partial Q$ consists of a finite number of $C^{3}$-smooth non-selfintersecting curves $\Gamma_{i}$, $i=1,2, \ldots, p$, which may be either closed or have common end-points.

Billiard in $Q$ is the dynamical system which corresponds to the motion of a material point inside $Q$ by inertia with elastic reflections at the boundary.

We consider the framing of each $\Gamma_{i}$ by unit normal vectors $n(q), q \in \Gamma_{i}$, directed inside $Q$. As a result the curvature of each $\Gamma_{i}$ takes a definite sign. Dispersed billiards are billiards for which all $\Gamma_{i}$ have a strictly positive curvature (see [1]).

Let $M$ be the unit tangent bundle over $Q, \pi$ is the natural projection of $M$ onto $Q$. Preimage $\pi^{-1}(q)=S^{1}(q), q \in Q$ consists of unit vectors which are tangent to $Q$ at $q \in Q . \quad M$ is the three-dimensional open manifold with the boundary $\partial M=\bigcup_{i=1}^{p} \pi^{-1}\left(\Gamma_{i}\right)=\bigcup_{i=1}^{p} \partial M_{i}$. On every $\partial M_{i}$ one can introduce natural coordinates $(r, \varphi)$ where $r$ is the parameter of length on every $\Gamma_{i}$ and $\varphi$ is the angle between $x$ and $n(q), q=\pi(x)$. Let

$$
\begin{aligned}
M_{1} & =\{x \in \partial M:(x, n(q)) \geqq 0, q=\pi(x)\}, \quad M_{1}^{(i)}=M_{1} \cap \partial M_{i} \\
S_{0} & =\{x \in \partial M:(x, n(q))=0, q=\pi(x)\}, \\
M_{2} & =\bigcup_{i \neq j} \pi^{-1}\left(\Gamma_{i} \cap \Gamma_{j}\right), \quad M_{s}=S_{0} \cup M_{2} .
\end{aligned}
$$

* Dedicated to the memory of Rufus Bowen 
Fig. 1

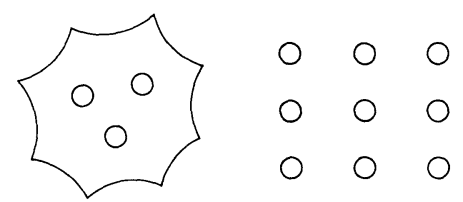

The set $M_{s}$ is called the set of singular points of the boundary. For simplicity we shall restrict ourselves to the case when $M_{2}=\emptyset$. The general case needs obvious modifications.

Liouville measure on $M$ takes the form $d \mu=d q d \omega$, where $d q$ is the usual Lebesque measure on the plane, $d \omega$ is the Haar measure on the circle $S^{(1)}(q)$ $=\pi^{-1}(q)$. One-parameter group of shifts along the trajectories of the billiard is denoted by $\left\{S^{t}\right\},-\infty<t<\infty$. It is wellknown that $\left\{S^{t}\right\}$ preserves the measure $\mu$.

Let $\tau(x), x \in M_{1}$, be the nearest positive moment of reflection of the trajectory from the boundary. It is easy to see that $\tau(x)<\infty$ and one can define the transformation of $M_{1}$ into itself by the formula $T x=S^{\tau(x)-0}, x \in M_{1} . T$ preserves the measure $v$ where $d v=$ const $\cos \varphi d r d \varphi$. Here the const does not depend on $\Gamma_{i}$ and can be chosen in such a way that $v$ will be the normed measure. Using the language of ergodic theory one can say that the flow $\left\{S^{t}\right\}$ is represented as a special flow built with the help of the automorphism $T$ of the base space $M_{1}$ and the function $\tau(x)$. The main aim of this and subsequent papers is to investigate the ergodic and stochastic properties of $T$.

We shall assume that $\tau(x)$ is uniformly bounded from above, i.e. for some constant $C$ we have $\tau(x) \leqq C$ for all $x$. Sometimes such billiards are called billiards with a finite horizon (see [7]). Our next assumption is that there are no trajectories of the flow $\left\{S^{t}\right\}$ which touch the boundary more than twice. It is easy to see that it is valid for general domains $Q$. Both these assumptions lead to some simplifications of a technical character in our future considerations.

Every subset $\pi^{-1}\left(\Gamma_{i}\right) \cap M_{1}=M_{1}^{(i)}$ is called a regular component of $M_{1}$. Suppose that we are given a curve $\delta$ which is described by a function $\varphi=\varphi(r)$ and belongs to a regular component of $M_{1}$. We shall call $\delta$ an increasing (decreasing) curve and denote it $\delta^{(u)}\left(\delta^{(s)}\right)$ if $\varphi(r)$ is piecewisely differentiable and one-sided derivatives satisfy the inequalities

$$
\begin{array}{r}
k^{(0)}(r)+\frac{\cos \varphi}{\frac{1}{k_{\min }^{(0)}}+\tau\left(T^{-1}(x(r, \varphi))\right)} \leqq \frac{d \varphi}{d r} \leqq k^{(0)}(r)+\frac{\cos \varphi}{\tau\left(T^{-1}(x(r, \varphi))\right)} \\
\cdot\left(-k^{(0)}(r)-\frac{\cos \varphi}{\tau(x(r, \varphi))} \leqq \frac{d \varphi}{d r} \leqq-k^{(0)}(r)-\frac{\cos \varphi}{\frac{1}{k_{\min }^{(0)}}+\tau(x(r, \varphi))}\right) .
\end{array}
$$

Here $k^{(0)}(r)$ is the curvature of the boundary at the point $r, x(r, \varphi) \in M_{1}$ is the point with the coordinates $r, \varphi, k_{\min }^{(0)}=\min _{r \in \partial Q} k^{(0)}(r),-\frac{\pi}{2} \leqq \varphi \leqq \frac{\pi}{2}$.

We shall use the following important property of increasing and decreasing curves (see [1]): if $\delta$ is an increasing (decreasing) curve and $T\left(T^{-1}\right)$ is continuous on $\delta$ then $T \delta\left(T^{-1} \delta\right)$ is again an increasing (decreasing) curve. 
Let $x \in M_{1}$ be an inner point. We shall call a $C^{1}$-curve $\gamma, x \in \gamma$, a stable manifold (an unstable manifold) of the point $x$ if $T^{i}$ is continuous on $\gamma$ for all $i>0(i<0)$ and the length $\left(T^{i} \gamma\right) \rightarrow 0$ as $i \rightarrow \infty(i \rightarrow-\infty)$. In [1] it is shown that for almost every $x$ there exist stable and unstable manifolds, which are decreasing and increasing curves correspondingly. We shall use the notations $\gamma^{(s)}(x), \gamma^{(u)}(x)$ for maximal smooth components of stable and unstable manifolds of $x$ and call them local stable transversal fibers (1.s.t.f.) and local unstable transversal fibers (1.u.t.f.).

In $[1,6]$ it is also shown that $\gamma^{(s)}(x), \gamma^{(u)}(x)$ can be represented as solutions of ordinary differential equations. For $\gamma^{(s)}(x)$ the differential equation takes the form

$$
\frac{d \varphi}{d r}=\kappa^{(s)}(x(\varphi, r)) \cos \varphi-k^{(0)}(x)
$$

where $x=(r, \varphi) \in M_{1}, k^{(0)}(x)$ is the curvature of the boundary at the point $r$ and $\kappa^{(s)}(x)$ is a continuous fraction

$$
\kappa^{(s)}(x)=\frac{1}{-\tau_{1}+\frac{1}{-\frac{2 k_{1}^{(0)}(x)}{\cos \varphi_{1}(x)}+\frac{1}{-\tau_{2}+\frac{1}{-\frac{2 k_{2}^{(0)}(x)}{\cos \varphi_{2}(x)}+\frac{1}{-\tau_{3}+\ldots}}}}}
$$

Here $\tau_{i}$ is the interval between $i$-th and $(i-1)$-th collisions, $\tau_{i}>0, k_{i}^{(0)}(x)$ is the curvature of the boundary at the point of $i$-th collision, $\varphi_{i}(x)$ is the angle of incidence at the point of $i$-th reflection.

The equation for $\gamma^{(u)}(x)$ can be written in an analogous way. If $A \subset M_{1}$ then $\gamma_{A}^{(s)}(x)=\gamma^{(s)}(x) \cap A, \gamma_{A}^{(u)}(x)=\gamma^{(s)}(x) \cap A$.

Let $\gamma_{1}^{(s)}, \gamma_{2}^{(s)}$ be two 1.s.t.f. Subsets $A C \gamma_{1}^{(s)}, B C \gamma_{2}^{(s)}$ are called canonically isomorphic if for every $x \in A$ the intersection $\gamma^{(u)}(x) \cap \gamma_{2}^{(s)}$ is not empty and consists of a point belonging to $B$ and vice versa. In a similar way one can define the canonical isomorphism between subsets of $\gamma_{1}^{(u)}, \gamma_{2}^{(u)}$. The canonical isomorphism is absolutely continuous, i.e. it transforms a measure on $\gamma_{1}^{(s)}$ which is equivalent to the length into a measure which is equivalent to the length on $\gamma_{2}^{(s)}$. The proof of this property is the same as the proof of an analogous property for hyperbolic systems (see $[2,5]$ ).

A subset $\mathscr{D} \subset M_{1}$ of positive measure will be called a parallelogram if for every pair of points $x^{\prime}, x^{\prime \prime} \in \mathscr{D}$ the intersection $\gamma^{(s)}\left(x^{\prime}\right) \cap \gamma^{(u)}\left(x^{\prime \prime}\right)$ is not empty and consists precisely of one point also belonging to $\mathscr{D}$ (see [2-4] for the analogous definition in the case of hyperbolic dynamical systems). If $\mathscr{D}$ is a parallelogram than all $\gamma_{\mathscr{D}}^{(s)}(x)$, $x \in \mathscr{D}$, are canonically isomorphic. The same statement is true for all $\gamma_{\mathscr{D}}^{(u)}(x)$. Moreover, every parallelogram can be constructed in the following way. We take an arbitrary $x_{0} \in \mathscr{D}$ and $\gamma_{\mathscr{D}}^{(s)}\left(x_{0}\right), \gamma_{\mathscr{D}}^{(u)}\left(x_{0}\right)$. Then

$$
\mathscr{D}=\bigcup_{x \in \gamma_{\mathscr{D}}^{(s)}\left(x_{0}\right), y \in \gamma_{\mathscr{D}}^{(u)}\left(x_{0}\right)} \gamma^{(s)}(y) \cap \gamma^{(u)}(x) \text {. }
$$

Let $\eta$ be a finite or countable partition of $M_{1}$ whose elements are parallelograms, $\eta=\left(\mathscr{D}_{1}, \mathscr{D}_{2}, \ldots\right), \mathscr{D}_{i} \cap \mathscr{D}_{j}=\emptyset, i \neq j, v\left(M_{1}-\bigcup_{i} \mathscr{D}_{i}\right)=0$. If $x \in \mathscr{D}_{i}$ we shall write $\gamma_{\eta}^{(u)}(x)$, $\gamma_{\eta}^{(s)}(x)$ instead of $\gamma_{\mathscr{D}_{i}}^{(u)}(x), \gamma_{\mathscr{D}_{i}}^{(s)}(x)$. 
Definition 1 (see $[2,3]$ ). The partition $\eta$ is called a Markov partition if for almost every $x \in M_{1}, x \in \mathscr{D}_{i}, T x \in \mathscr{D}_{j_{1}}, T^{-1} x \in \mathscr{D}_{j_{2}}$

$$
T\left(\gamma_{\eta}^{(s)}(x)\right) \subseteq \gamma_{\eta}^{(s)}(T x), \quad T^{-1}\left(\gamma_{\eta}^{(u)}(x)\right) \subseteq \gamma_{\eta}^{(u)}\left(T^{-1} x\right) .
$$

The main result of this paper is the following theorem.

Theorem. Under the conditions formulated above there exists a countable Markov partition.

It is sufficient to construct a Markov partition $\xi$ for $T^{m}$ for some $m>0$ because $\xi \vee T \xi \vee \ldots \vee T^{m-1} \xi$ will be a Markov partition for $T$.

It is well-known that Markov partitions play a key role in investigations of ergodic properties of hyperbolic systems (see [2] and monographs by Bowen [3] and Ruelle [4]). The main reason is that using Markov partitions one can rewrite the measure $v$ in a form of a limit Gibbs state with nice properties of the corresponding potential. We shall see that in our case it is also possible. Using this approach we investigate in the next paper, which is in fact the second part of this paper, statistical properties such as decay of correlations, central limit theorem and Donsker's invariance principle for dispersed billiards.

The proof of the main theorem consists of three steps. In Sect. 3 we construct an initial partition which has already some Markov properties. In Sect. 4 we construct an auxiliary pre-Markov finite partition (see the next section for definitions). In Sect. 5 we construct the final Markov partition. In Sect. 6 we investigate symbolic dynamics and some other properties of $T$ with respect to the Markov partition.

\section{Some Preliminary Definitions and Facts Concerning the Theory of Dispersed Billiards}

In the beginning we shall consider various partitions of $M_{1}$ whose elements have a sufficiently simple form. We shall call polygons open connected domains in $M_{1}$ belonging to a single regular component of the boundary whose boundary consists of a finite number of $C^{1}$-curves. Each of these curves is either decreasing or increasing or belongs to $S_{0}$. We shall call quadrilaterals such polygons for which the boundary consists of two increasing (left and right) and two decreasing (upper and lower) curves. If $C$ is a polygon then $\Gamma_{1}^{(u)}(C)\left(\Gamma_{1}^{(s)}(C)\right)$ is the part of the boundary $\partial C$ of $C$ consisting of increasing (decreasing) curves, $\Gamma_{0}(C)=\partial C \cap S_{0}$.

Let $S_{k}=T^{k} S_{0},-\infty<k<\infty$. It follows easily from [1] that $S_{k}$ for $k>0(k<0)$ consists of increasing (decreasing) curves whose end-points belong to $\bigcup_{0 \leqq k^{\prime} \leqq k} S_{k^{\prime}}$ $\left(\bigcup_{k \leqq k^{\prime} \leqq 0} S_{k^{\prime}}\right)$ (see Fig. 2).

For a polygon $C$ we denote $\Gamma^{(u)}(C)\left(\Gamma^{(s)}(C)\right)$ the set of components of $\Gamma_{1}^{(u)}(C)\left(\Gamma_{1}^{(s)}(C)\right)$ not belonging to $\bigcup_{k>0} S_{k}\left(\bigcup_{k<0} S_{k}\right)$. Suppose that we are given a partition $\xi$ whose elements are polygons, i.e. $\xi=\left(C_{1}, C_{2}, \ldots\right), C_{i} \cap C_{j}=\emptyset, i \neq j$, $v\left(M_{1}-\bigcup_{i} C_{i}\right)=0 . \quad$ We put $\Gamma_{1}^{(s)}(\xi)=\bigcup_{i} \Gamma_{1}^{(s)}\left(C_{i}\right), \quad \Gamma^{(s)}(\xi)=\bigcup_{i} \Gamma^{(s)}\left(C_{i}\right), \quad \Gamma_{1}^{(u)}(\xi)$ $=\bigcup_{i} \Gamma_{1}^{(u)}\left(C_{i}\right), \Gamma^{(u)}(\xi)=\bigcup_{i} \Gamma^{(u)}\left(C_{i}\right), \Gamma_{0}(\xi)=\bigcup_{i} \Gamma_{0}\left(C_{i}\right)=S_{0}$. 

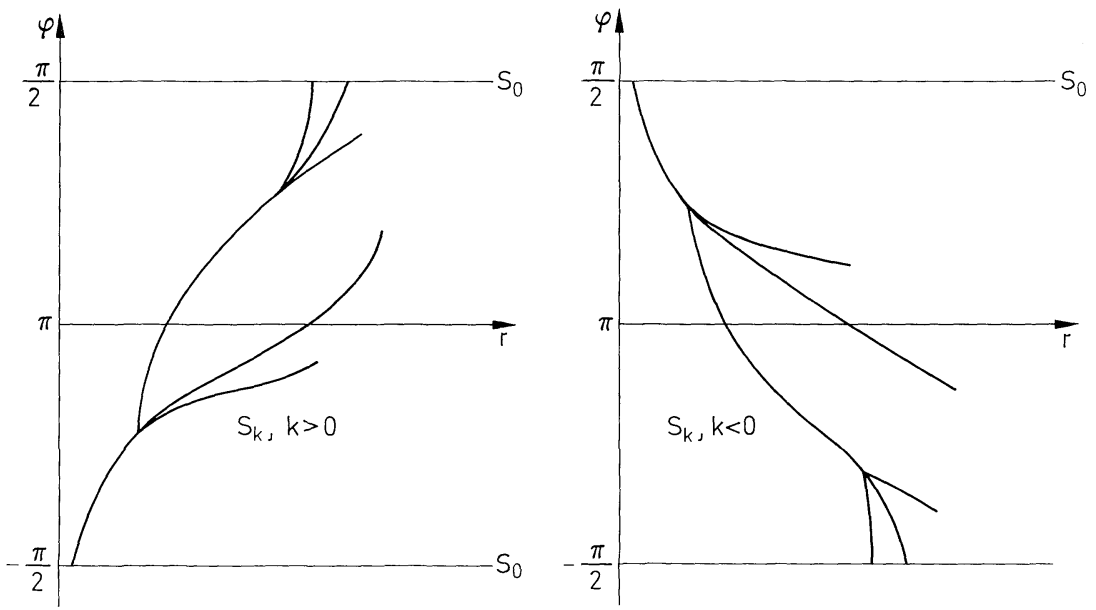

Fig. 2

Definition 2. A partition $\xi$ is a pre-Markov partition if for some $m>0$

$$
\Gamma^{(s)}\left(T^{m} \xi\right) \subset \Gamma^{(s)}(\xi), \quad \Gamma^{(u)}\left(T^{-m} \xi\right) \subset \Gamma^{(u)}(\xi) .
$$

We shall construct pre-Markov partitions in Sect. 4. Now we shall discuss in more detail properties of discontinuity of $T^{m}, m>0$. It is easy to see that $T^{m}$ is discontinuous on $\bigcup_{k=1}^{m} S_{-k}$. A point $x \in S_{k} \cap S_{l}$ is called a double point if $k \cdot l \geqq 0$. Geometrically the double points generate trajectories which touch the boundary twice either for $t \geqq 0$ or for $t \leqq 0$. The index ind $(x)$ of a double point $x$ is equal by definition to $\max (|k|,|l|) \cdot \operatorname{sgn} k$ if $k \neq 0$ or $l$ if $k=0$. The set of double points for which $k \leqq$ ind $(x) \leqq l$ is denoted by $W_{k}^{l}$.

Let us denote by $S_{k, i}, i \in I_{k}$, smooth components of $S_{k}$. Their end-points are double points. We shall consider the structure of $S_{-m}$ in a neighbourhood of a double point $z \in S_{-m}$, ind $(z) \geqq-m$. The following four possibilities for such double points can arise.

a) $z \in S_{0}$ (see Fig. 3).

$\left.\mathrm{b}_{1}\right) z \notin S_{0}, z \in S_{-m-1, i_{1}} \cap S_{-k, j} \cap S_{-m, i_{2}}$ (see Fig. 4).

The point $z$ is an end-point of $S_{-m, i_{2}}, i_{2} \in I_{-m}$, an inner point of $S_{-k, j}, j \in I_{-k}$, $0<k<m$, and an end-point of $S_{-m-1, i_{1}}, i_{1} \in I_{-m-1}$. The mapping $T_{1}=T^{m}$ is continuous on $S_{-k, j}$ and on $S_{-m-1, i_{1}}$ till $z$. On the curve $S_{-m, i_{2}}$ the mapping $T_{1}$ is discontinuous at $z$.

$\left.\mathrm{b}_{2}\right) z \notin S_{0}, z \in S_{-m+1, i_{1}} \cap S_{-k, j} \cap S_{-m, i_{2}}$.

For $T^{-1} z$ we have $b_{1}$ ). Here $z$ is an inner point of $S_{-k, j}$ and a common endpoint of $S_{-m+1, i_{1}}, S_{-m, i_{2}}$. The mapping $T_{1}$ is continuous on $S_{-k, j}, S_{-m, i_{1}}$. On the curve $S_{-m+1, i_{2}}$ it is continuous outside $z$.

$\left.\mathrm{b}_{3}\right) z \notin S_{0}, z \in S_{-m, i_{1}} \cap S_{-m, i_{2}} \cap S_{-m-1, i_{3}}$ (see Fig. 6).

Here $z$ is an end-point of $S_{-m, i_{2}}$ and an inner point of $S_{-m, i_{1}} . T_{1}$ is continuous on $S_{-m, i_{1}}$ and discontinuous on $S_{-m, i_{2}}$ at $z$. 

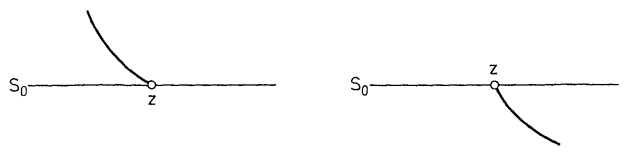

Fig. 3

Fig. 4
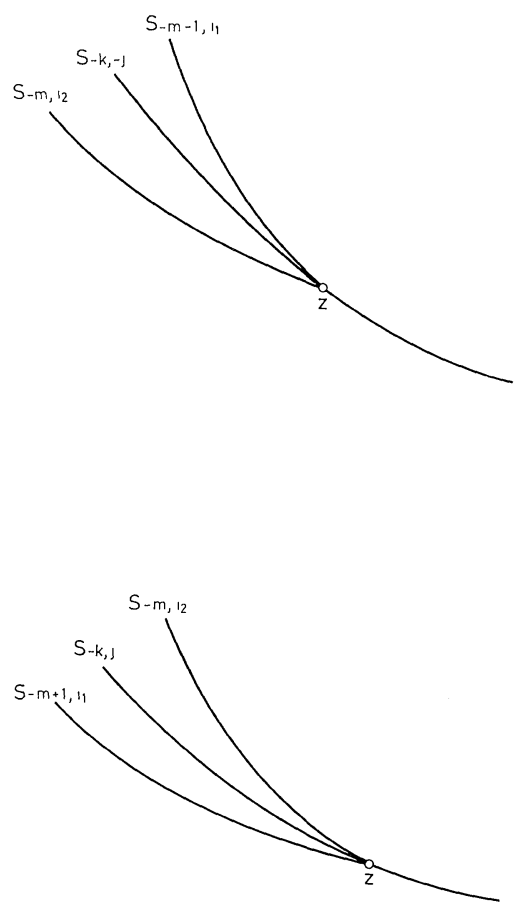

Fig. 5

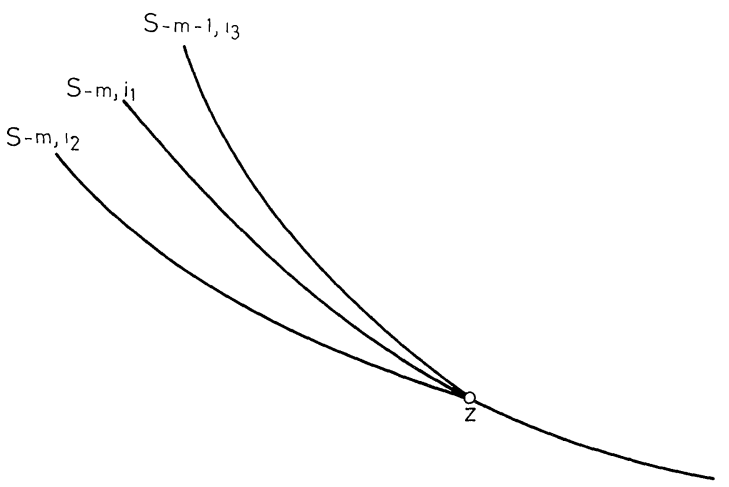

Fig. 6 
Now we shall introduce a constant of geometrical nature. Suppose that $\delta^{(u)}\left(\delta^{(s)}\right)$ is an increasing (decreasing) curve and $T\left(T^{-1}\right)$ is continuous on $\delta^{(u)}\left(\delta^{(s)}\right)$. It follows from [1] that $l\left(\delta^{(u)}\right) \leqq l\left(T \delta^{(u)}\right), l\left(\delta^{(s)}\right) \leqq l\left(T^{-1} \delta^{(s)}\right), l$ is the length of the curve ${ }^{1}$. We put

$$
\Lambda_{\min }=\min \left(\inf _{\delta^{(u)}} \frac{l\left(T \delta^{(u)}\right)}{l\left(\delta^{(u)}\right)}, \inf _{\delta^{(s)}} \frac{l\left(T^{-1} \delta^{(s)}\right)}{l\left(\delta^{(s)}\right)}\right) .
$$

It follows easily from [1] that $1<\Lambda_{\min }<\infty$, see also [6].

By a neighbourhood of a regular point $x \in M_{1}$ we shall mean a circle with a center at $x$ and a semi-circle centered at $x$ for $x \in S_{0}$. If the radius of the circle is $\alpha$ we shall write $O_{\alpha}(x)$. The closure of a set $A$ is denoted by $\bar{A}$.

\section{Construction of an Initial Partition}

We shall construct in this section an initial partition $\xi_{0}$ of the phase space $M_{1}$ which will be transformed in the next section into a pre-Markov partition. The elements of $\xi_{0}$ will be polygons, the boundary of $\xi_{0}$ will contain $\bigcup_{|k| \leqq m} S_{k}$. Elements of $\xi_{0}$ which have components of the boundary belonging to $\bigcup_{|k| \leqq m} S_{k}$ will be called adjacent polygons. Other elements will be called non-adjacent polygons.

The construction of $\xi_{0}$ consists of several steps. To begin with we shall construct vertices of adjacent polygons (in fact in Lemma 1) lying on $\bigcup_{|k| \leqq m} S_{k}$. Next we construct the sides of adjacent polygons intersecting $\bigcup_{|k| \leqq m} S_{k}$. This part of the boundary will already have a Markov property. Namely if $\delta_{0}^{(u)}$ is a smooth component of $\Gamma^{(u)}\left(\xi_{0}\right)$ intersecting $\bigcup_{-m \leqq k \leqq 0} S_{k}$ at $x$ then $T_{1} x=T^{m} x$ is contained in a curve $\delta_{1}^{(u)} \subset \Gamma^{(u)}\left(\xi_{0}\right)$ and $T_{1} \delta_{0}^{(u)} \supseteqq \delta_{1}^{(u)}$. The same property will hold for $\delta_{0}^{(s)}$. Also all non-adjacent polygons will be quadrilaterals.

Lemma 1. Let a natural number $m$ be given. For all sufficiently small $\varepsilon$ there exist finite sets $\mathscr{P}_{\varepsilon}\left(S_{j}\right) \subset S_{j},|j| \leqq m$, such that

1) $\mathscr{P}_{\varepsilon}\left(S_{j}\right) \subseteq T \mathscr{P}_{\varepsilon}\left(S_{j-1}\right)$ for $j \leqq 0 ; \mathscr{P}_{\varepsilon}\left(S_{j}\right) \subseteq T^{-1} \mathscr{P}_{\varepsilon}\left(S_{j+1}\right)$ for $j \geqq 0$;

2) dist $\left(z^{\prime}, z^{\prime \prime}\right) \geqq C_{1} \varepsilon$ for arbitrary $z^{\prime}, z^{\prime \prime} \in \bigcup_{|j| \leqq m} \mathscr{P}_{\varepsilon}\left(S_{j}\right)$;

3) $\operatorname{dist}\left(z, \bigcup_{|j| \leqq m} \mathscr{P}_{\varepsilon}\left(S_{j}\right)-z\right) \leqq C_{2} \varepsilon$ for arbitrary $z \in \bigcup_{|j| \leqq m} \mathscr{P}_{\varepsilon}\left(S_{j}\right)$;

4) $\operatorname{dist}\left(z, \bigcup_{|j| \leqq m} \mathscr{P}_{\varepsilon}\left(S_{j}\right)\right) \leqq C_{2} \varepsilon$ for arbitrary $z \in \bigcup_{|j| \leqq m} S_{j}$;

5) for every end-point $z$ of each $S_{k, i},|k| \leqq m, i \in I_{k}$, there exists a neighbourhood $O^{(1)}(z) \supseteqq O_{\varepsilon^{5 / 6}}(z)$ such that the set $\bigcup_{|j| \leqq m} \mathscr{P}_{\varepsilon}\left(S_{j}\right) \cap O^{(1)}(z)$ has a special form which will be described later and which will be called a right form.

Here and later $C_{i}, i=1,2, \ldots$ are constants which do not depend on $m, \varepsilon$ but depend only on the geometrical characteristics of the billiard.

The proof consists of several steps.

$1^{\circ}$. Let us take $W_{1}^{m}\left(S_{0}\right)=W_{1}^{m} \cap S_{0}$ and $T^{-m} W_{1}^{m}\left(S_{0}\right) \subseteq S_{-m}$. For every $z \in T^{-m} W_{1}^{m}\left(S_{0}\right)$ we consider its $\varepsilon^{2 / 3}$-neighbourhood $O_{\varepsilon^{2 / 3}}(z)$ and $\tilde{O}_{\varepsilon^{2 / 3}}(z)=O_{\varepsilon^{2 / 3}}(z)$

1 Here and further under the length of a curve we mean the length of its projection onto $r$-axis 
$\cap S_{-m}$. It is clear that if $\varepsilon=\varepsilon(m)$ is sufficiently small then either $\tilde{O}_{\varepsilon^{2 / 3}}(z)$ is a simple open curve, $\left(\tilde{O}_{\varepsilon^{2 / 3}}(z) \subset S_{-m, i}\right.$ for some $\left.i \in I_{-m}\right)$ or we have the case $\left.b_{3}\right)$. For different $z$ and $s, 0 \leqq s \leqq m$, the neighbourhoods $T^{s} O_{\varepsilon^{2 / 3}}(z)$ do not intersect if $\varepsilon$ is small enough. Also $T^{m} \tilde{O}_{\varepsilon^{2 / 3}}(z) \subset S_{0}$.

Let $\mathscr{N}_{\varepsilon}(z)$ be a maximal subset of $\tilde{O}_{\varepsilon^{2 / 3}}(z)$ consisting of points for which the mutual distances are not less than $\varepsilon$ if we disallow the case $b_{3}$ ). We can construct inductively a sequence of sets $\mathcal{N}_{\varepsilon}\left(T^{s} z\right), T^{s} z \in \mathcal{N}_{\varepsilon}\left(T^{s} z\right) \subset T^{s} \tilde{O}_{\varepsilon^{2 / 3}}(z), 0 \leqq s \leqq m$, with the following properties:

1) $T \mathscr{N}_{\varepsilon}\left(T^{s} z\right) \supseteqq \mathscr{N}_{\varepsilon}\left(T^{s+1} z\right), 0 \leqq s<m$;

2) the mutual distances of points of $\mathscr{N}_{\varepsilon}\left(T^{s} z\right)$ are not less than $\varepsilon$;

3) if $\mathscr{N}_{\varepsilon}\left(T^{s} z\right)$ is chosen then $\mathscr{N}_{\varepsilon}\left(T^{s+1} z\right)$ is a maximal set of points satisfying 1$)$ and 2).

If a double point $z$ is such that we have the case $b_{3}$ ) then we consider

$$
O_{\varepsilon^{2 / 3}}(z) \cap S_{-m}=\tilde{O}_{\varepsilon^{2 / 3}}(z)
$$

and a maximal subset $\mathscr{N}_{\varepsilon}(z), z \in \mathscr{N}_{\varepsilon}(z) \subset \tilde{O}_{\varepsilon^{2 / 3}}(z)$ such that

1) $\operatorname{dist}\left(z^{\prime}, z^{\prime \prime}\right) \geqq \varepsilon$ for arbitrary $z^{\prime}, z^{\prime \prime} \in \mathscr{N}_{\varepsilon}(z)$;

2) if for every $z^{\prime} \in \mathcal{N}_{\varepsilon}(z)$ we construct a straight segment $\delta\left(z^{\prime}\right)$ passing through $z^{\prime}$ at an angle with the $r$-axis equal to $\varphi^{(u)}\left(z^{\prime}\right)$ the end-points of which belong to $\partial O_{\varepsilon^{2 / 3}}(z)$ then $\operatorname{dist}\left(z^{\prime}, \bigcup_{z^{\prime \prime} \in \mathcal{N}_{\varepsilon}(z)-z^{\prime}} \delta\left(z^{\prime \prime}\right)\right) \geqq \varepsilon$. Here $\operatorname{tg} \varphi^{(u)}(z)=\kappa^{(u)}(z)$.

We can construct now a sequence of sets $\mathscr{N}_{\varepsilon}\left(T^{s} z\right), 1 \leqq s \leqq m$, with the same properties 1,2 , as above.

$2^{\circ}$. Let $z \in S_{-k} \cap S_{-m} \cap S_{-m-1}, 0<k<m$ [case $\left.\left.b_{1}\right)\right]$. We consider the neighbourhood $T^{m-k} O_{\varepsilon^{2 / 3}}\left(T^{k-m} z\right)$. For sufficiently small $\varepsilon$ we have

$$
T^{m-k} O_{\varepsilon^{2 / 3}}\left(T^{k-m} z\right) \supseteqq O_{\varepsilon^{5 / 6}}(z) .
$$

In $1^{\circ}$ we have constructed $\mathscr{N}_{\varepsilon}(z)$,

$$
z \in \mathscr{N}_{\varepsilon}(z) \subset S_{-k, j} \subset S_{-k} .
$$

Let us take now for every $y \in \mathscr{N}_{\varepsilon}(z)$ a straight line segment passing through $y$ and having the angle $\varphi^{(u)}(y)$ with the $r$-axis with the end-points belonging to $\partial\left(T^{m-k} O_{\varepsilon^{2 / 3}}\left(T^{k-m} z\right)\right)$. We denote by $\mathscr{N}_{\varepsilon}^{\prime}(z)$ a maximal subset of

$$
T^{m-k} O_{\varepsilon^{2 / 3}}\left(T^{k-m} z\right) \cap S_{-m}
$$

with the following properties:

1) $\operatorname{dist}\left(z^{\prime}, z^{\prime \prime}\right) \geqq \varepsilon / 3$ for arbitrary $z^{\prime}, z^{\prime \prime} \in \mathcal{N}_{\varepsilon}^{\prime}(z)$;

2) for every $z^{\prime} \in \mathcal{N}_{\varepsilon}^{\prime}(z)$ its distance to the straight line segments constructed above is not less than $\varepsilon / 3$.

It is easy to see that if $\varepsilon$ is sufficiently small then there is at least one point of $\mathcal{N}_{\varepsilon}^{\prime}$ between every two neighbouring segments (see Fig. 7).

$3^{\circ}$. Let $z \in S_{-k} \cap S_{-m} \cap S_{-m+1}, 0<k<m-1$ [case $\left.b_{2}\right)$ ]. In this case point $T^{-1} z$ was considered in $2^{\circ}$. Let us construct a maximal subset $\mathscr{N}_{\varepsilon}^{\prime}(z) \subseteq T \mathscr{N}_{\varepsilon}^{\prime}\left(T^{-1} z\right)$ for which

1) $\operatorname{dist}\left(z^{\prime}, z^{\prime \prime}\right) \geqq \varepsilon / 9 \Lambda_{\min }^{2}$ for arbitrary $z^{\prime}, z^{\prime \prime} \in \mathscr{N}_{\varepsilon}^{\prime}(z)$;

2) if we construct for every $y \in \mathscr{N}_{\varepsilon}(z)$ a straight line segment with a slope angle $\varphi^{(u)}(y)$ passing through $y$ and having the end-points belonging to 
Fig. 7

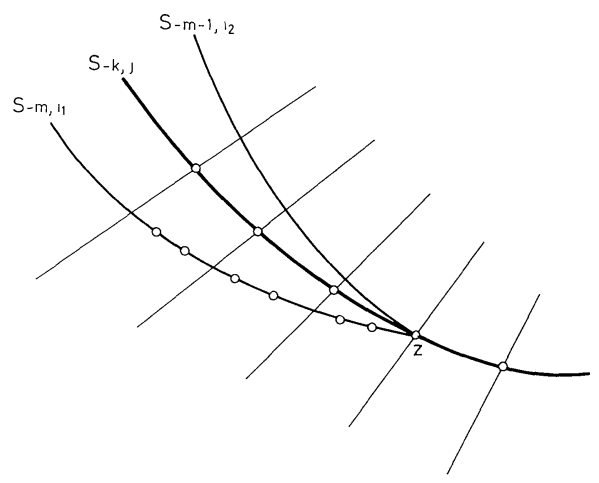

$\partial\left(T^{m-k} O_{\varepsilon^{2 / 3}}\left(T^{k-m} z\right)\right.$ then the distance between $\mathscr{N}_{\varepsilon}^{\prime}(z)$ and these segments is not less than $\varepsilon / 9 \Lambda_{\text {min }}^{2}$.

As before, it follows from the maximality of $\mathscr{N}_{\varepsilon}^{\prime}(z)$ that there is at least one point of $\mathscr{N}_{\varepsilon}^{\prime}(z)$ between two neighbouring segments.

Now we construct a maximal subset

for which

$$
\mathcal{N}_{\varepsilon}^{\prime \prime}(z) \subset S_{-m} \cap T^{m-k} O_{\varepsilon^{2 / 3}}\left(T^{k-m} z\right)
$$

1) $\operatorname{dist}\left(z^{\prime}, z^{\prime \prime}\right) \geqq \varepsilon / 9 \Lambda_{\min }^{2}$ for arbitrary $z^{\prime}, z^{\prime \prime} \in \mathcal{N}_{\varepsilon}^{\prime \prime}(z)$,

2) let us construct for every $y \in \mathscr{N}_{\varepsilon}(z) \cup \mathcal{N}_{\varepsilon}^{\prime}(z)$ a straight segment at an angle $\varphi^{(u)}(z)$ the end-points of which belong to $\partial\left(T^{m-k} O_{\varepsilon^{2 / 3}}\left(T^{k-m} z\right)\right)$; then the distance from any $x \in \mathscr{N}_{\varepsilon}^{\prime \prime}(z)$ to these segments is not less than $\varepsilon / 9 \Lambda_{\text {min }}^{2}$. We shall denote

$$
\mathscr{N}_{\varepsilon}^{\prime \prime \prime}(z)=\mathscr{N}_{\varepsilon}(z) \cup \mathscr{N}_{\varepsilon}^{\prime}(z) \cup \mathcal{N}_{\varepsilon}^{\prime \prime}(z)
$$

As before it follows from the maximality of $\mathscr{N}_{\varepsilon}^{\prime \prime \prime}(z)$ that there is at least one point of $\mathcal{N}_{\varepsilon}^{\prime \prime}(z)$ between every two neighbouring segments in 2).

$4^{\circ}$. For every $z \in S_{-k} \cap S_{-m} \cap S_{-m+1}, 0<k<m-1$, [case b $\left.b_{2}\right)$ we take $\mathscr{N}_{\varepsilon}(z)$, $\mathscr{N}_{\varepsilon}^{\prime}(z), \mathscr{N}_{\varepsilon}^{\prime \prime}(z)$ and

$$
\mathscr{N}_{\varepsilon}^{\prime \prime \prime}(z)=\mathscr{N}_{\varepsilon}(z) \cup \mathcal{N}_{\varepsilon}^{\prime}(z) \cup \mathscr{N}_{\varepsilon}^{\prime \prime}(z)
$$

Then we construct inductively a sequence of sets $\mathscr{N}_{\varepsilon}\left(T^{i} z\right), \mathscr{N}_{\varepsilon}^{\prime}\left(T^{i} z\right), \mathscr{N}_{\varepsilon}^{\prime \prime}\left(T^{i} z\right)$, $\mathcal{N}_{\varepsilon}^{\prime \prime \prime}\left(T^{i} z\right), 0 \leqq i \leqq k-1$, such that

1) $\mathscr{N}_{\varepsilon}^{\prime}\left(T^{i} z\right) \subseteq T \mathcal{N}_{\varepsilon}^{\prime}\left(T^{i-1} z\right), \mathscr{N}_{\varepsilon}^{\prime \prime}\left(T^{i} z\right) \subseteq T \mathcal{N}_{\varepsilon}^{\prime \prime}\left(T^{i-1} z\right)$;

2) $\mathscr{N}_{\varepsilon}^{\prime}\left(T^{i} z\right), \mathscr{N}_{\varepsilon}^{\prime \prime}\left(T^{i} z\right)$ satisfy conditions 1$\left.), 2\right)$ of $3^{\circ}$,

3) for given $\mathscr{N}_{\varepsilon}^{\prime}\left(T^{i-1} z\right), \mathscr{N}_{\varepsilon}^{\prime \prime}\left(T^{i-1} z\right)$ the sets $\mathscr{N}_{\varepsilon}^{\prime}\left(T^{i} z\right), \mathscr{N}_{\varepsilon}^{\prime \prime}\left(T^{i} z\right)$ are maximal set satisfying 1) and 2).

In case $b_{2}$ ) the point $T^{k} z \in S_{0}$. There is a closed semi-neighbourhood

$$
O_{1} \subset T^{m-k} O_{\varepsilon^{2 / 3}}\left(T^{-m+k} z\right)
$$

containing a part of a curve $S_{-m, i}$ where the mapping $T^{k}$ is continuous. We can construct a subset

$$
\mathcal{N}_{\varepsilon}^{\prime \prime \prime}\left(T^{k} z\right) \subset T^{k}\left(O_{1} \cap\left(S_{-k} \cup S_{-m}\right)\right)
$$

with the same properties 1)-3). 
Now let us consider $k+1$ for $k<m$. It is easy to see that if

$$
\begin{aligned}
& S^{\prime}=S_{-m} \cap T^{m-k} O_{\varepsilon^{2 / 3}}\left(T^{-m+k} z\right), \\
& S^{\prime \prime}=S_{-m+1} \cap T^{m-k} O_{\varepsilon^{2 / 3}}\left(T^{-m+k} z\right)
\end{aligned}
$$

then $T^{k+1} S^{\prime}, T^{k} S^{\prime \prime}$ are parts of a smooth curve $S_{-m+k+1, j}, j \in I_{-m+k+1}$ for which $T^{k+1} z$ is a common end-point. We construct $\mathscr{N}_{\varepsilon}^{\prime \prime \prime}\left(T^{k+1} z\right)$,

$$
T^{k+1} z \in \mathcal{N}_{\varepsilon}^{\prime \prime \prime}\left(T^{k+1} z\right) \subset T^{k+1} S^{\prime} \cup T^{k} S^{\prime \prime}
$$

satisfying 1)-3). Next we construct a sequence of sets $\mathscr{N}_{\varepsilon}^{\prime \prime \prime}\left(T^{i} z\right), k+1<i \leqq m$ satisfying 1)-3).

$5^{\circ}$. For every double point $z \in \bigcup_{k=0}^{m} S_{-k},-m \leqq$ ind $(z)<0$ we have already constructed a neighbourhood or a semi-neighbourhood for $z \in S_{-m} \cap S_{0}$ which is denoted by $O^{(1)}(z)$ and which contains $O_{\varepsilon^{5 / 6}}(z)$ if $\varepsilon$ is sufficiently small. In $O^{(1)}(z)$ we have constructed the set $\mathscr{N}_{\varepsilon}^{\prime \prime \prime}(z)^{2}$ with the following properties:

1) $\operatorname{dist}\left(z^{\prime}, z^{\prime \prime}\right) \geqq \varepsilon / 9 \Lambda_{\min }^{2}$ for arbitrary $z^{\prime}, z^{\prime \prime} \in \mathcal{N}_{\varepsilon}^{\prime \prime \prime}(z)$;

2) if we construct a straight segment passing through $z^{\prime} \in \mathscr{N}_{\varepsilon}^{\prime \prime \prime}(z)$ at an angle $\varphi^{(u)}(z)$ with the end-points belonging to $\partial O^{(1)}(z)$ then the mutual distances between these segments are not less than $\varepsilon / 18 \Lambda_{\min }^{2}$;

3) $\mathscr{N}_{\varepsilon}^{\prime \prime \prime}(z) \cong T \mathcal{N}_{\varepsilon}^{\prime \prime \prime}\left(T^{-1} z\right)$,

4) $\mathscr{N}_{\varepsilon}^{\prime \prime \prime}(z)$ is a maximal set satisfying 1$\left.)-3\right)$.

The mapping $T$ contracts all curves $S_{-k, i}, i \in I_{k}$. Therefore

5) $\operatorname{dist}\left(z^{\prime}, \mathscr{N}_{\varepsilon}^{\prime \prime \prime}(z)-z^{\prime}\right) \leqq C_{2} \varepsilon$ for every $z^{\prime} \in \mathscr{N}_{\varepsilon}^{\prime \prime \prime}(z)$.

If 1)-5) are valid for some constants not depending on $m, \varepsilon$ instead of $1 / 9 \Lambda_{\text {min }}^{2}$, $C_{2}$ etc., we shall say that the set has a right form in the neighbourhood $O^{(1)}(z)$.

Now we take

$$
S_{-m}-\bigcup_{\left.z \in\left(\underset{0<k \leqq m}{\bigcup} \bigcup_{(W-m}^{-k} \cap S_{-m}\right)\right)} O^{(1)}(z)
$$

and construct a maximal subset $\mathscr{N}_{\varepsilon}^{(-m)} \subseteq S_{-m}-\bigcup_{z} O^{(1)}(z)$ such that $\operatorname{dist}\left(z^{\prime}, z^{\prime \prime}\right) \geqq \varepsilon$ for all $z^{\prime}, z^{\prime \prime} \in \mathscr{N}_{\varepsilon}^{(-m)}$. Next we construct inductively a sequence of sets $\mathscr{N}_{\varepsilon}^{(-i)}$, $0 \leqq i<m$, for which dist $\left(z^{\prime}, z^{\prime \prime}\right) \geqq \varepsilon$ for all $z^{\prime}, z^{\prime \prime} \in \mathscr{N}_{\varepsilon}^{(-i)}, T \mathscr{N}_{\varepsilon}^{(-i-1)} \supseteqq \mathscr{N}_{\varepsilon}^{(-i)}$ and $\mathscr{N}_{\varepsilon}^{(-i)}$ is a maximal set satisfying these conditions.

$$
\begin{gathered}
\text { Let } \mathscr{N}_{\varepsilon}=\bigcup_{0 \leqq i \leqq m} \mathscr{N}_{\varepsilon}^{(-i)} \cup \bigcup_{z} \mathscr{N}_{\varepsilon}^{\prime \prime \prime}(z) \text { and } \mathscr{N}_{\varepsilon}\left(S_{-k}\right)=\mathscr{N}_{\varepsilon} \cap S_{-k}, 1 \leqq k \leqq m, \\
\mathscr{N}_{\varepsilon}^{-}\left(S_{0}\right)=\mathscr{N}_{\varepsilon} \cap S_{0}
\end{gathered}
$$

where the union is taken over all double points $z \in W_{-m}^{-1}$.

Changing $T$ on $T^{-1}$ and vice versa we get an analogous sequence of sets $\mathscr{N}_{\varepsilon}\left(S_{m}\right), \mathscr{N}_{\varepsilon}\left(S_{m-1}\right), \ldots, \mathscr{N}_{\varepsilon}\left(S_{1}\right), \mathcal{N}_{\varepsilon}^{+}\left(S_{0}\right)$. Certainly it can happen that $\mathscr{N}_{\varepsilon}^{-}\left(S_{0}\right)$ $\neq \mathscr{N}_{\varepsilon}^{+}\left(S_{0}\right)$ and some points of these sets are too close to each other. We take

$$
\mathscr{N}_{\varepsilon}^{-}\left(S_{0}\right) \cup \mathcal{N}_{\varepsilon}^{+}\left(S_{0}\right)
$$

2 If $z=T^{i} z_{1}, 0 \leqq i \leqq m$, and for $z_{1}$ we have the case $\left.b_{3}\right)$ then $\mathscr{N}_{\varepsilon}^{\prime \prime \prime}(z)=\mathscr{N}_{\varepsilon}(z)$ 
and construct a maximal subset

$$
\mathscr{N}_{\varepsilon}\left(S_{0}\right) \subseteq \mathscr{N}_{\varepsilon}^{-}\left(S_{0}\right) \cup \mathscr{N}_{\varepsilon}^{+}\left(S_{0}\right)
$$
consisting of points the mutual distances of which are not less than $\frac{C_{1}^{\prime} \varepsilon}{9 \Lambda_{\min }^{2}}$ with
$C_{1}^{\prime}>\frac{1}{2}, C_{1}^{\prime}<1$. For every

$$
z \in \mathscr{N}_{\varepsilon}^{-}\left(S_{0}\right)-\mathscr{N}_{\varepsilon}\left(S_{0}\right)
$$

one can find $z^{\prime} \in \mathscr{N}_{\varepsilon}\left(S_{0}\right)$ for which

$$
\operatorname{dist}\left(z, z^{\prime}\right)<C_{1}^{\prime} \varepsilon\left(9 \Lambda_{\min }^{2}\right)^{-1} \text {. }
$$

This point $z^{\prime} \in \mathscr{N}_{\varepsilon}^{+}\left(S_{0}\right)$ because mutual distances of points of $\mathscr{N}^{-}\left(S_{0}\right)$ are not less than $C_{1}^{\prime} \varepsilon\left(9 \Lambda_{\min }^{2}\right)^{-1}$.

For every such pair $\left(z, z^{\prime}\right)$ we consider $\left(T^{-1} z, T^{-1} z^{\prime}\right),\left(T^{-2} z, T^{-2} z^{\prime}\right), \ldots$, $\left(T^{-k} z, T^{-k} z^{\prime}\right)$ where $k \leqq m$ is the least number for which

$$
\operatorname{dist}\left(T^{-k} z, T^{-k} z^{\prime}\right) \geqq C_{1}^{\prime} \varepsilon\left(9 \Lambda_{\min }^{2}\right)^{-1}
$$

If such a $k$ does not exist, we put $k=m$. Now we exclude the points $z, T^{-1} z, \ldots, T^{-k} z$ from set $\bigcup_{i=0}^{k} \mathscr{N}_{\varepsilon}\left(S_{-i}\right)$ and instead of them we include points $z^{\prime}, T^{-1} z^{\prime}, \ldots, T^{-k} z^{\prime}$. One can show that there does not exist a point

$$
z^{\prime \prime} \in \bigcup_{i=0}^{m} \mathscr{N}_{\varepsilon}\left(S_{-i}\right)
$$

for which

$$
\operatorname{dist}\left(z^{\prime \prime}, T^{-i} z^{\prime}\right) \leqq \frac{C_{1}^{\prime} \varepsilon}{2}\left(9 \Lambda_{\min }^{2}\right)^{-1}
$$

for some $i, 0 \leqq i \leqq k$. Further we consider points $T^{-k-1} z^{\prime}, \ldots, T^{-m} z^{\prime}$. Suppose that for some $l \geqq k+1$ one can find

$$
z^{\prime \prime} \in \bigcup_{i=l}^{m} \mathscr{N}_{\varepsilon}\left(S_{-i}\right)
$$

for which

$$
\operatorname{dist}\left(z^{\prime \prime}, T^{-l} z^{\prime}\right) \leqq \frac{C_{1}^{\prime} \varepsilon}{2}\left(9 \Lambda_{\min }^{2}\right)^{-1}
$$

In this case we take the least $k_{1}>l$ for which

$$
\operatorname{dist}\left(T^{-k_{1}} z^{\prime \prime}, T^{-l-k_{1}} z^{\prime}\right) \geqq C_{1}^{\prime} \varepsilon\left(9 \Lambda_{\min }^{2}\right)^{-1}
$$

(if such a $k_{1}$ does not exist we put $k_{1}=m-l$ ). It is worthwhile to mention that $T^{-1}$ expands the curves $S_{-k, i}$. Now we exclude from the set $\bigcup_{i=l}^{l+k} \mathscr{N}_{\varepsilon}\left(S_{-i}\right)$ the points $z^{\prime \prime}$, $T^{-1} z^{\prime \prime}, \ldots, T^{-k_{1}} z^{\prime \prime}$ and we include instead the points $T^{-l} z^{\prime}, T^{-l-1} z^{\prime}, \ldots T^{s-l-k_{1}} z^{\prime}$. 
Such a procedure will be performed until $m$ is reached with all points of

$$
\mathscr{N}_{\varepsilon}^{-}\left(S_{0}\right)-\mathscr{N}_{\varepsilon}\left(S_{0}\right)
$$

In every neighbourhood $O^{(1)}(z)$ the new set will have a right form because we replace a point by a new point for which the distance from the original one is not more than $\frac{1}{2} C_{1}^{\prime} \varepsilon\left(9 \Lambda_{\min }^{2}\right)^{-1}$. Thus we get the desired set. Q.E.D.

Let $\mathscr{P}_{\varepsilon}$ be the set constructed in Lemma 1. For $z \in S_{-m} \cap \mathscr{P}_{\varepsilon}$ lying outside all $O^{(1)}\left(z_{1}\right)$ [see 5) in Lemma $1, z_{1}$ is a double point] we construct a straight segment $\delta^{(u)}(z)$ passing through $z$ at an angle $\varphi^{(u)}(z)$ such that the distance from $z$ to its endpoints are equal to $C_{3} \varepsilon$. Here again $C_{3}$ is a constant not depending on $m, \varepsilon$ but depending only on geometrical properties of the billiard. Later we shall suppose that $C_{3}$ is large enough compared with $A_{\min }, C_{1}, C_{2}$ etc. From $z \notin \bigcup_{z_{1}} O^{(1)}\left(z_{1}\right)$ it follows that $T^{m-1}$ is continuous on $\delta^{(u)}(z)$. For every $i, 0 \leqq i<m$, such that $T^{i} z \in \mathscr{P}_{\varepsilon}\left(S_{-m+i}\right)$ we construct an increasing curve $\delta^{(u)}\left(T^{i} z\right)$ for which

1) $\delta^{(u)}\left(T^{i} z\right) \subseteq T \delta^{(u)}\left(T^{i-1} z\right)$

2) the distances from $T^{i} z$ to the end-points of $\delta^{(u)}\left(T^{i} z\right)$ are equal to $C_{3} \varepsilon$.

Now we shall make an analogous construction in neighbourhoods $O^{(1)}\left(z_{1}\right)$ of double points $z_{1} \in S_{-m}$. Suppose that $z_{1} \notin S_{0}{ }^{3}$. For every

$$
y \in O^{(1)}\left(z_{1}\right) \cap \bigcup_{k=0}^{m} \mathscr{P}_{\varepsilon}\left(S_{-k}\right)
$$

we construct a maximal straight segment $\delta_{1}^{(u)}(y)$ passing through $y$ at an angle $\varphi^{(u)}(y)$. In order to define a segment $\delta^{(u)}(y) \subseteq \delta_{1}^{(u)}(y)$ we shall consider various possibilities.

$a_{1} . \delta_{1}^{(u)}(y)$ intersects all components of $\bigcup_{k=1}^{m} S_{-k}$ lying in $O^{(1)}\left(z_{1}\right)$. The total number of these components is not more than three [see cases $\left.\left.b_{1}\right)-b_{3}\right)$ ]. The length of each inner component of $\delta_{1}^{(u)}(y)$ bounded by points of $\bigcup_{k=0}^{m} S_{-k}$ is not greater than $2 C_{3} \varepsilon$; we shall define in this case $\delta^{(u)}(y)$ so that it contains both inner components and the lengths of boundary components are equal to $C_{3} \varepsilon$ where boundary component has only one of its end-points on $\bigcup_{k=0}^{m} S_{-k}$.

$\mathrm{a}_{2}$. An inner component of $\delta_{1}^{(u)}(y)$ which is adjacent to $y$ has a length less than $2 C_{3} \varepsilon$, but the next inner component has a length bigger than $2 C_{3} \varepsilon$; in this case $\delta^{(u)}(y)$ contains an inner component which is adjacent to $y$ and two boundary components the lengths of which are equal to $C_{3} \varepsilon$.

$\mathrm{a}_{3}$. Both inner components $\delta_{1}^{(u)}(y)$ have a length bigger than $2 C_{3} \varepsilon$; in this case $\delta^{(u)}(y)$ has only boundary components the lengths of which are equal to $C_{3} \varepsilon$. All the cases are drawn in Fig. 8.

Assume that for $z \in \mathscr{P}_{\varepsilon}\left(S_{-m}\right)$ we have constructed curve $\delta^{(u)}(z)$ and $T^{i}, 0 \leqq i<k$, are continuous on $\delta^{(u)}(z)$. For

$$
z_{1}=T^{i} z \in \mathscr{P}_{\varepsilon}\left(S_{-m+i}\right)
$$

we construct inductively curves $\delta^{(u)}\left(T^{i} z\right)$ in such a way, that

1) $\delta^{(u)}\left(z_{1}\right) \subseteq T \delta^{(u)}\left(T^{-1} z\right)$

3 The case $z_{1} \in S_{0}$ is considered below 
Fig. 8

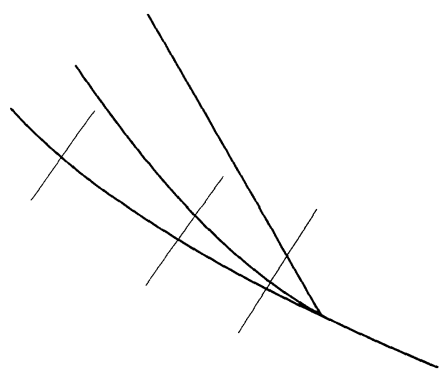

2) $\delta^{(u)}\left(z_{1}\right)$ has the same properties as described in $\mathrm{a}_{1}-\mathrm{a}_{3}$ above except the fact that it is a straight segment.

So far we have constructed $\delta^{(u)}(z)$ for some $z \in \bigcup_{k=1}^{m} \mathscr{P}_{\varepsilon}\left(S_{-k}\right)$. In those cases $T$ was continuous on $\delta^{(u)}\left(T^{-1} z\right)$ and $\delta^{(u)}(z) \subseteq T \delta^{(u)}\left(T^{-1} z\right)$. Now we expand our construction to $z_{1} \in \mathscr{P}_{\varepsilon}\left(S_{0}\right)$ or $z_{1} \in O^{(1)}(z), z \in S_{-m} \cap S_{0}$. In the first case we take the maximal component $\delta_{1}^{(u)}$ of $\delta^{(u)}\left(T^{-1} z_{1}\right)$ containing $T^{-1} z_{1}$ where $T$ is continuous and we put $\delta^{(u)}\left(z_{1}\right)=T \delta_{1}^{(u)}$. In the second case we construct a straight segment passing through $z_{1}$ at an angle $\varphi^{(u)}\left(z_{1}\right)$ and either the distances from $z_{1}$ to both end-points are equal to $C_{3} \varepsilon$ or only one of them is equal to $C_{3} \varepsilon$ while the other end-point belongs to $S_{0}$. In the same way as above we construct inductively $\delta^{(u)}\left(T^{i} z_{1}\right)$ for those $i$ for which $T$ is continuous on $\delta^{(u)}\left(T^{i-1} z_{1}\right)$. Next we make the analogous construction of curves $\delta^{(s)}(z)$ for $z \in \bigcup_{k=1}^{m} \mathscr{P}_{\varepsilon}\left(S_{k}\right)$.

Now we come to a situation where $z \in O^{(1)}\left(z_{0}\right) \cap \mathscr{P}_{\varepsilon}\left(S_{-1}\right)$ for a double point $z_{0} \in W_{-2 m}^{-1} \cap S_{-1}$ and $T$ is discontinuous on $\delta^{(u)}(z)$. Here $\delta^{(u)}(z)$ consists of two components $\delta_{1}^{(u)}(z), \delta_{2}^{(u)}(z)$ and $T$ is continuous on each of them. We assume that their common end-point belongs to $\delta_{1}^{(u)}(z)$ and $T$ is continuous on $\delta_{1}^{(u)}(z)$ including the end-points. Three possibilities can arise:

1) $T z \in S_{0}$;

2) $z \in \delta_{1}^{(u)}(z), T z \in \bigcup_{k=1}^{m} S_{-k}$ and $T, T^{2}$ are continuous on $\delta_{1}^{(u)}(z)$;

3) $z \in \delta_{2}^{(u)}(z), T z \in \bigcup_{k=1}^{m} S_{-k}$ and $T$ is continuous on $\delta_{2}^{(u)}(z)$.

In case 1) we define $\delta^{(u)}\left(T^{2} z\right)$ only if $T^{2} z \in \mathscr{P}_{\varepsilon}\left(S_{1}\right)$. In case 2) we define $\delta^{(u)}\left(T^{2} z\right)$ only if $T^{2} z \in \bigcup_{k=1}^{m} \mathscr{P}_{\varepsilon}\left(S_{-k}\right)$. In case 3) we define $\delta^{(u)}(T z)$ only if

$$
T z \in \bigcup_{k=1}^{m} \mathscr{P}_{\varepsilon}\left(S_{-k}\right)
$$

We consider

$$
T^{2} \delta_{1}^{(u)}(z) \cup T \delta_{2}^{(u)}(z)
$$

It is easy to see that

$$
T^{2} \delta_{1}^{(u)}(z) \cup T \delta_{2}^{(u)}(z)
$$


Fig. 9

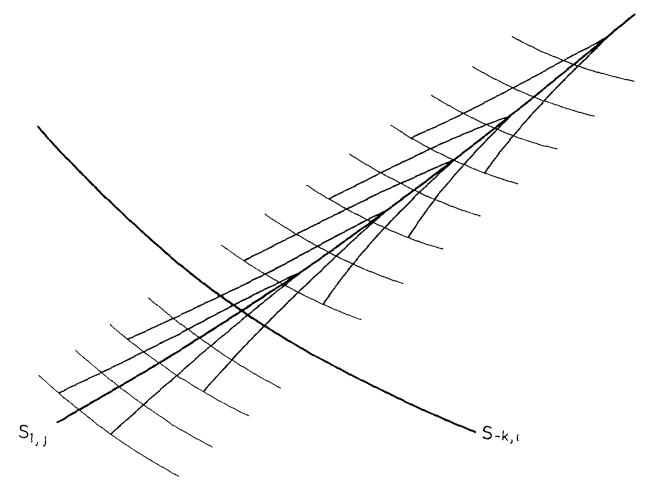

is a connected set consisting of two smooth components having a common endpoints $x$, lying on $S_{1}$. Each of components is an increasing curve. Now we recall that we have "the fence" of curves $\delta^{(s)}(y)$ along $S_{1}$. Let $z_{1}=T^{2} z$ in cases 1),2) and $z_{1}=T z$ in case 3 ). We put

$$
\delta^{(u)}\left(z_{1}\right)=\delta_{1}^{(u)}\left(z_{1}\right) \cup \delta_{2}^{(u)}\left(z_{1}\right),
$$

where $\delta_{1}^{(u)}\left(z_{1}\right) \subseteq T^{2} \delta_{1}^{(u)}(z), \delta_{2}^{(u)}\left(z_{1}\right) \leqq T \delta^{(u)}$ are smooth increasing curves,

$$
\delta_{1}^{(u)}\left(z_{1}\right) \cap \delta_{2}^{(u)}\left(z_{1}\right)=T^{2} \delta_{1}^{(u)}(z) \cap T \delta_{2}^{(u)}(z)
$$

and other end-points of these curves belong to a curve $\delta^{(s)}(y)$. Moreover,

$\left.\mathrm{b}_{1}\right)$ if $\delta_{1}^{(u)}\left(z_{1}\right) \cap \delta^{(s)}\left(y_{1}\right) \neq \emptyset$ for some $y_{1} \in \mathscr{P}_{\varepsilon}\left(S_{1}\right)$ then $\delta_{2}^{(u)}\left(z_{1}\right) \cap \delta^{(s)}\left(y_{1}\right) \neq \emptyset$ and the distances from $\delta_{1}^{(u)}\left(z_{1}\right) \cap \delta^{(s)}\left(y_{1}\right), \delta_{2}^{(u)}\left(z_{1}\right) \cap \delta^{(s)}\left(y_{1}\right)$ to $y_{1}$ are not more than $\frac{1}{2} C_{3} \varepsilon$;

$\left.\mathrm{b}_{2}\right)$ each $\delta^{(s)}(y)$ cannot contain the end-points of more than one $\delta^{(u)}\left(z_{1}\right)$;

$\left.\mathrm{b}_{3}\right) \delta^{(u)}\left(z_{1}\right)$ intersects with at least two $\delta^{(s)}(y)$.

$\mathrm{b}_{4}$ ) for some $\gamma, 0<\gamma<1$, which depend on geometrical properties of the billiard, the lengths of $\delta_{1}^{(u)}(z), \delta_{2}^{(u)}(z)$ are not less than $\gamma C_{3} \varepsilon$. If $\delta_{1}^{(u)}\left(z_{1}\right)$ or $\delta_{2}^{(u)}\left(z_{1}\right)$ is cut by $\bigcup_{k=1}^{m} S_{-k}$ onto two pieces then the length of each piece is not less, than $\gamma C_{3} \varepsilon$.

If $z \in O^{(1)}\left(z_{0}\right), z_{0} \in S_{-m} \cap S_{0}$, then $\delta^{(u)}(z)$ is a straight segment for which at least one half $\delta_{1}^{(u)}(z)$ has the length $C_{3} \varepsilon$ while the other half $\delta_{2}^{(u)}(z)$ can be less. We put

$$
\delta^{(u)}(T z)=T \delta_{2}^{(u)}(z) \cup \delta_{1}^{(u)}(T z)
$$

if the length of $T \delta_{2}^{(u)}(z)$ less than $\gamma C_{3} \varepsilon$ and $\delta_{1}^{(u)}(T z) \subset T \delta_{1}^{(u)}(z)$ is chosen in such a way that $\left.\left.b_{1}\right)-b_{4}\right)$ are valid. If the length of $T \delta_{2}^{(u)}(z)$ is bigger than $\gamma C_{3} \varepsilon$ we make the same construction as above.

The form of $\delta^{(u)}(z)$ is drawn on Fig. 9. The set of $\delta^{(u)}(z)$ along $S_{1}$ looks like a firtree.

Let $z \in \bigcup_{k=1}^{m} \mathscr{P}_{\varepsilon}\left(S_{-k}\right) \cup \bigcup_{k=1}^{m} T^{k} \mathscr{P}_{\varepsilon}\left(S_{0}\right)$ be such that $\delta^{(u)}(z)$ is not defined yet but for some $i, 0 \leqq i<m, \delta^{(u)}\left(T^{-i} z\right)$ is already defined and consists of two components $\delta_{1}^{(u)}\left(T^{-i} z\right), \bar{\delta}_{2}^{(u)}\left(T^{-i} z\right)$ and $T^{i}$ is continuous on $\delta^{(u)}\left(T^{-i} z\right)$. We put in this case $\delta^{(u)}(z)$ $=T^{i} \delta^{(u)}\left(T^{-i} z\right)$. Now $\delta^{(u)}(z)$ is defined for all $z \in \bigcup_{k=1}^{m} \mathscr{P}_{\varepsilon}\left(S_{-k}\right)$ and for some 
Fig. 10

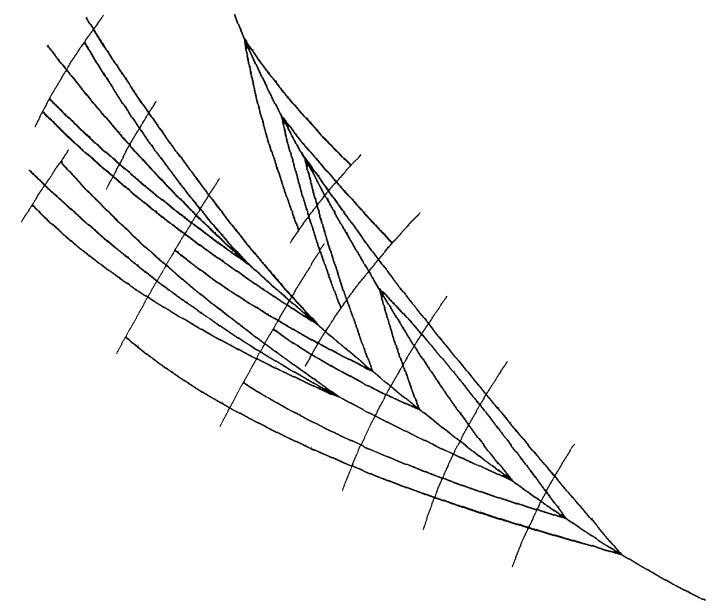

$z \in \mathscr{P}_{\varepsilon}\left(S_{0}\right) \cup \bigcup_{k=1}^{m} T^{k} \mathscr{P}_{\varepsilon}\left(S_{0}\right)$. If $\delta^{(u)}(z)$ is defined and $z \notin \mathscr{P}_{\varepsilon}\left(S_{-m}\right)$ then $T \delta^{(u)}\left(T^{-1} z\right) \supseteqq \delta^{(u)}(z)$. Now we continue our construction for the rest of $z \in \mathscr{P}_{\varepsilon}\left(S_{0}\right)$. For such $z \delta^{(u)}\left(T^{-1} z\right)$ is already defined but $T$ is discontinuous on $\delta^{(u)}\left(T^{-1} z\right)$, consisting of two smooth components. We take the maximal connected component $\delta_{3}^{(u)}\left(T^{-1} z\right) \subset \delta^{(u)}\left(T^{-i} z\right)$ where $T$ is continuous and $T^{-1} z$ is one of its end-points. We put $\delta^{(u)}(z)=T \delta_{3}^{(u)}$ $\cdot\left(T^{-1} z\right)$. Now $\delta^{(u)}(z)$ is defined for all $z \in \bigcup_{k=0}^{m} \mathscr{P}_{\varepsilon}\left(S_{k}\right)$ and some $z \in \bigcup_{k=1}^{m} T^{k} \mathscr{P}_{\varepsilon}\left(S_{0}\right)$. If $z$ is such that $\delta^{(s)}(z), \delta^{(s)}(T z)$ are defined then $T^{-1} \delta^{(s)}(T z) \supseteqq \delta^{(s)}(z)$. Quite similarly we can define $\delta^{(s)}(z)$ for all $z \in \bigcup_{k=0}^{m} \mathscr{P}_{\varepsilon}\left(S_{k}\right)$ and some $z \in \bigcup_{k=1}^{m} T^{-k} \mathscr{P}_{\varepsilon}\left(S_{0}\right)$. If $z$ is such that $\delta^{(s)}(z), \delta^{(s)}(T z)$ are defined then $T^{-1} \delta^{(s)}(T z) \supseteqq \delta^{(s)}(z)$. Moreover, we can perform our construction in such a way that if $\delta^{(u)}\left(z_{1}\right) \cap \delta^{(s)}\left(z_{2}\right) \neq \emptyset$ then $\delta^{(u)}\left(T^{-k} z_{1}\right)$ $\cap \delta^{(s)}\left(T^{-k} z_{2}\right) \neq \emptyset$ for such $k$ for which $\delta^{(u)}\left(T^{-k} z_{1}\right), \delta^{(s)}\left(T^{-k} z_{2}\right)$ are defined.

We continue our construction and intend to define $\delta^{(u)}(z)$ for the rest of $z \in \bigcup_{k=1}^{m} T^{k} \mathscr{P}_{\varepsilon}\left(S_{0}\right)$. Suppose that $z \in T \mathscr{P}_{\varepsilon}\left(S_{0}\right) \subset \mathscr{P}_{\varepsilon}\left(S_{1}\right)$. We must consider only the case when $\delta^{(u)}\left(T^{-2} z\right)$ is defined, consists of two smooth increasing components and $T$ is discontinuous on $\delta^{(u)}\left(T^{-2} z\right)$. In other words $\delta^{(u)}\left(T^{-2} z\right)=\delta_{1}^{(u)}\left(T^{-2} z\right) \cup \delta_{2}^{(u)}\left(T^{-2} z\right)$ and $T, T^{2}$ is continuous on $\delta_{1}^{(u)}\left(T^{-2} z\right), \quad T$ is continuous on $\delta_{2}^{(u)}\left(T^{-2} z\right)$ and $T^{2} \delta_{1}^{(u)}\left(T^{-2} z\right) \cup T \delta_{2}^{(u)}\left(T^{-2} z\right)$ is a connected set, consisting of four smooth increasing components. We put

$$
\delta^{(u)}(z)=T^{2} \delta_{1}^{(u)}\left(T^{-2} z\right) \cup T \delta_{2}^{(u)}\left(T^{-2} z\right)
$$

(see Fig. 10). If $z \in T^{k} \mathscr{P}_{\varepsilon}\left(S_{0}\right)$ for some $k \leqq m$ and $T^{-k+1} z \in T \mathscr{P}_{\varepsilon}\left(S_{0}\right) \subset S_{1}, \delta^{(u)}\left(T^{-k+1} z\right)$ is already defined and consists of four smooth increasing components we put $\delta^{(u)}(z)$ $=T^{k-1} \delta^{(u)}\left(T^{-k+1} z\right)$. In the same way we define $\delta^{(s)}(z)$ for the rest of $z \in \bigcup_{k=1}^{m} T^{-k} \mathscr{P}_{\varepsilon}\left(S_{0}\right)$. Now we can formulate the results of our considerations in the form of the following lemma. 
Lemma 2. For every

$$
z \in \bigcup_{k=0}^{m} \mathscr{P}_{\varepsilon}\left(S_{-k}\right) \cup \bigcup_{k=1}^{m} T^{k} \mathscr{P}_{\varepsilon}\left(S_{0}\right) \quad\left(z \in \bigcup_{k=0}^{m} \mathscr{P}_{\varepsilon}\left(S_{k}\right) \cup \bigcup_{k=1}^{m} T^{-k} \mathscr{P}_{\varepsilon}\left(S_{0}\right)\right)
$$

one can construct a connected set $\delta^{(u)}(z), z \in \delta^{(u)}(z)\left(\delta^{(s)}(z), z \in \delta^{(s)}(z)\right)$ consisting of $j \leqq 4$ smooth increasing (decreasing) components in such a way that

1) if $\delta^{(u)}(z)\left(\delta^{(s)}(z)\right)$ consists of one increasing (decreasing) component then distances from $z$ to the end-points of $\delta^{(u)}(z)\left(\delta^{(s)}(z)\right)$ are equal to $C_{3} \varepsilon$; for others $j$ the length of each smooth component is not less than $\gamma C_{3} \varepsilon$;

2) for

$$
\begin{gathered}
z \in \bigcup_{k=0}^{m} \mathscr{P}_{\varepsilon}\left(S_{-k}\right) \cup \bigcup_{k=1}^{m-1} T^{k} \mathscr{P}_{\varepsilon}\left(S_{0}\right) \quad\left(z \in \bigcup_{k=0}^{m} \mathscr{P}_{\varepsilon}\left(S_{k}\right) \cup \bigcup_{k=1}^{m-1} T^{-k} \mathscr{P}_{\varepsilon}\left(S_{0}\right)\right) \\
T \delta^{(u)}(z) \supseteqq \delta^{(u)}(T z) \quad\left(T^{-1} \delta^{(s)}(z) \supseteqq \delta^{(s)}\left(T^{-1} z\right)\right)
\end{gathered}
$$

if $T\left(T^{-1}\right)$ is continuous on $\delta^{(u)}(z)\left(\delta^{(s)}(z)\right)$; if $T\left(T^{-1}\right)$ is discontinuous on $\delta^{(u)}(z)$ $\left(\delta^{(s)}(z)\right)$ then

$$
\delta^{(u)}(T z) \leqq T \delta^{(u)}(z) \cup T^{2} \delta^{(u)}(z) \quad\left(\delta^{(s)}\left(T^{-1} z\right) \subseteq T^{-1} \delta^{(s)}(z) \cup T^{-2} \delta^{(s)}(z)\right) ;
$$

3) the end-points of $\delta^{(u)}(z)\left(\delta^{(s)}(z)\right)$, for which $j \geqq 2$, belong to a $\delta^{(s)}\left(z_{1}\right)\left(\delta^{(u)}\left(z_{1}\right)\right)$ with $j=1$,

$$
z_{1} \in \bigcup_{k=0}^{m} \mathscr{P}_{\varepsilon}\left(S_{k}\right) \quad\left(z_{1} \in \bigcup_{k=0}^{m} \mathscr{P}_{\varepsilon}\left(S_{-k}\right)\right)
$$

$\delta^{(s)}\left(z_{1}\right)\left(\delta^{(u)}\left(z_{1}\right)\right)$ does not contain the end-points of more than two $\delta^{(u)}(z)\left(\delta^{(s)}(z)\right)$; if $\delta^{(u)}(z) \cap \delta^{(s)}\left(z_{1}\right) \neq \emptyset \quad\left(\delta^{(s)}(z) \cap \delta^{(u)}\left(z_{1}\right) \neq \emptyset\right)$ then the distance from $\delta^{(u)}(z) \cap \delta^{(s)}\left(z_{1}\right)$ $\left(\delta^{(s)}(z) \cap \delta^{(u)}\left(z_{1}\right)\right)$ to $z_{1}$ is not more than $\gamma C_{3} \varepsilon$;

4) every $\delta^{(s)}(z)\left(\delta^{(u)}(z)\right)$ with $j=1$ intersects at least two different $\delta^{(u)}(y)\left(\delta^{(s)}(y)\right)$ with $j>1$;

5) if $\delta^{(u)}(z) \cap \delta^{(s)}\left(z_{1}\right) \neq \emptyset$ and $0<k<m$ is such that $\delta^{(u)}\left(T^{-k} z\right), \quad \delta^{(s)}\left(T^{-k} z_{1}\right)$, $\left(\delta^{(u)}\left(T^{k} z\right), \delta^{(s)}\left(T^{k} z_{1}\right)\right)$ are defined then

$$
\delta^{(u)}\left(T^{-k} z\right) \cap \delta^{(s)}\left(T^{-k} z_{1}\right) \neq \emptyset \quad\left(\delta^{(u)}\left(T^{k} z\right) \cap \delta^{(s)}\left(T^{k} z_{1}\right) \neq \emptyset\right) .
$$

The property 2) shows that all $\delta^{(u)}(z)\left(\delta^{(s)}(z)\right)$ are uniquely defined via the construction by $\delta^{(u)}(y)\left(\delta^{(s)}(y)\right)$ for $y \in \mathscr{P}_{\varepsilon}\left(S_{-m}\right)\left(y \in \mathscr{P}_{\varepsilon}\left(S_{m}\right)\right)$ and by the sequence of sets $\mathscr{P}_{\varepsilon}\left(S_{k}\right)$ satisfying the properties of Lemma 1 .

Our construction is flexible and structurally stable in the following sense. There exists a constant $C_{4}=C_{4}\left(C_{1}, C_{2}, C_{3}\right)$ such that for any sets $\mathscr{P}_{\varepsilon}^{\prime}\left(S_{-m}\right) \subset S_{-m}$, $\mathscr{P}_{\varepsilon}^{\prime}\left(S_{m}\right) \subset S_{m}$ which are small deformations of $\mathscr{P}_{\varepsilon}\left(S_{-m}\right), \mathscr{P}_{i}\left(S_{m}\right)$ in the sense that for every $z \in \mathscr{P}_{\varepsilon}\left(S_{-m}\right)\left(z \in \mathscr{P}_{\varepsilon}\left(S_{m}\right)\right)$ one can find $z^{\prime} \in \mathscr{P}_{\varepsilon}^{\prime}\left(S_{-m}\right)\left(z^{\prime} \in \mathscr{P}_{\varepsilon}\left(S_{m}\right)\right)$ such that $\operatorname{dist}\left(z, z^{\prime}\right)$ $\leqq C_{4} \varepsilon$ and the correspondence $z \rightarrow z^{\prime}$ is one-to-one, we can take $\delta^{(u)}(z), z \in \mathscr{P}_{\varepsilon}^{\prime}\left(S_{-m}\right)$; $\delta^{(s)}(z), z \in \mathscr{P}_{\varepsilon}^{\prime}\left(S_{m}\right)$ and perform the whole construction and get the rest of $\delta^{(u)}(y)$, $\delta^{(s)}\left(y^{\prime}\right)$ with same properties. It is possible because one can construct the sets $\mathscr{P}_{\varepsilon}^{\prime}\left(S_{-k}\right) \quad\left(\mathscr{P}_{\varepsilon}^{\prime}\left(S_{k}\right)\right), \quad 0 \leqq k<m, \quad T \mathscr{P}_{\varepsilon}^{\prime}\left(S_{-k}\right) \supseteqq \mathscr{P}_{\varepsilon}^{\prime}\left(S_{-k+1}\right) \quad\left(T^{-1} \mathscr{P}_{\varepsilon}^{\prime}\left(S_{k}\right) \supseteqq \mathscr{P}_{\varepsilon}^{\prime}\left(S_{k-1}\right)\right) \quad$ for $0 \leqq k \leqq m$ and $\mathscr{P}_{\varepsilon}^{\prime}\left(S_{-k}\right)\left(\mathscr{P}_{\varepsilon}^{\prime}\left(S_{k}\right)\right)$ will be a small deformation of $\mathscr{P}_{\varepsilon}\left(S_{-k}\left(\mathscr{P}_{\varepsilon}\left(S_{k}\right)\right)\right.$. In other words, the set of $\delta^{(u)}(z), z \in \mathscr{P}_{\varepsilon}^{\prime}\left(S_{-m}\right)$ and $\delta^{(s)}(z), z \in \mathscr{P}_{\varepsilon}^{\prime}\left(S_{m}\right)$ is the defining set for the construction. 
Fig. 11

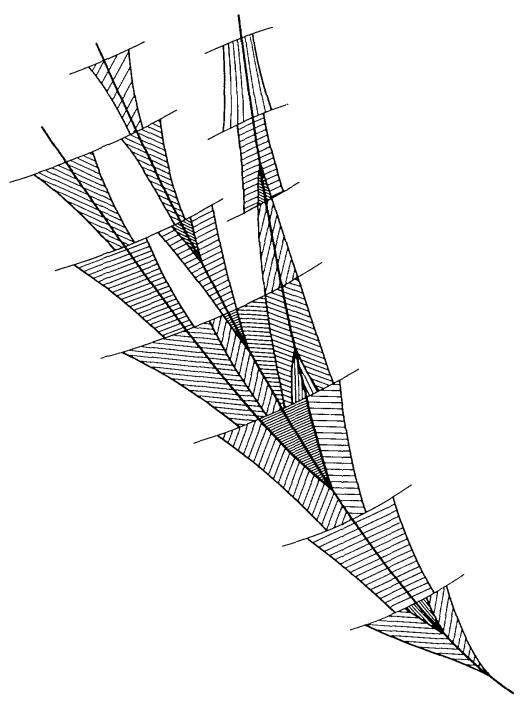

Let us return to the original situation and consider the set

$$
\bigcup_{|k| \leqq m} S_{k} \cup \bigcup_{z \in \bigcup_{k=0}^{m} \mathscr{P}_{\varepsilon}(S-k) \cup \bigcup_{i=1}^{m} T^{2} \mathscr{P}_{\varepsilon}\left(S_{0}\right)} \delta^{(u)}(z) \cup \bigcup_{z \in \bigcup_{k=0}^{m}} \bigcup_{\mathscr{P}_{\varepsilon}\left(S_{k}\right) \cup \bigcup_{i=1}^{m} T^{-\imath \mathscr{P}_{\varepsilon}\left(S_{0}\right)}} \delta^{(s)}(z)=S .
$$

In the neighbourhoods of $\bigcup_{|k| \leqq m} S_{k}$ there are maximal connected components $\mathscr{D}^{(0)}$ bounded by curves from $S$. It easy to see that each $\mathscr{D}^{(0)}$ is a polygon and can be triangle, quadrilateral or pentagon. The polygon $\mathscr{D}^{(0)}$ is called adjacent to $S_{k}$, $|k| \leqq m$ if a smooth component of the boundary $\partial \mathscr{D}^{(0)}$ belongs to $S_{k}$. Certainly, it can happen that $\mathscr{D}^{(0)}$ is adjacent to two $S_{k_{1}}, S_{k_{2}}, k_{1} \neq k_{2}$.

Definition 3. The union of all $\mathscr{D}^{(0)}$, adjacent to $S_{k}$, is called the necklace of $S_{k}$. It will be denoted by $\mathfrak{N}\left(S_{k}\right)$.

The form of the necklace in neighbourhoods of different types is drawn on the Fig. 11. Sometimes we shall write $\mathscr{D}_{i}^{(0)}(k)$ for $\mathscr{D}_{i}^{(0)}(k) \subset \mathfrak{N}\left(S_{k}\right)$. A polygon $\mathscr{D}^{(0)}$ for which $\partial \mathscr{D}^{(0)} \cap \bigcup_{|k| \leqq m} S_{k}=\emptyset$ is a quadrilateral. It follows easily from the fact that different $\delta^{(u)}$ or different $\delta^{(s)}$ do not intersect each other.

Now we shall formulate the next lemma.

Let $Q_{\varepsilon}^{\prime}$ be a maximal set with the following properties:

1) $\operatorname{dist}\left(z^{\prime}, z^{\prime \prime}\right) \geqq \varepsilon$ for arbitrary $z^{\prime}, z^{\prime \prime} \in Q_{\varepsilon}^{\prime}$;

2) $\operatorname{dist}\left(z, \cup \mathscr{D}^{(0)}\right) \geqq \varepsilon$ for $z \in Q_{\varepsilon}^{\prime}$.

Lemma 3. There exists such a set $Q_{\varepsilon}^{\prime \prime}$ that for every $z^{\prime \prime} \in Q_{\varepsilon}^{\prime \prime}$ one can find $z^{\prime} \in Q_{\varepsilon}^{\prime}$ for which $\operatorname{dist}\left(z^{\prime}, z^{\prime \prime}\right) \leqq C_{5} \varepsilon$, where $C_{5}=C_{5}\left(C_{1}, C_{2}, C_{3}, \Lambda_{\min }\right)$ is small enough and the correspondence $z^{\prime} \rightarrow z^{\prime \prime}$ is one-to-one. The new set $Q_{\varepsilon}^{\prime \prime}$ has the following properties:

1) for every $z^{\prime \prime} \in Q_{\varepsilon}^{\prime \prime}$ one can construct a straight segment $\delta^{(u)}\left(z^{\prime \prime}\right)\left(\delta^{(s)}\left(z^{\prime \prime}\right)\right)$ passing through $z^{\prime \prime}$ at an angle $\varphi^{(u)}\left(z^{\prime \prime}\right)\left(\varphi^{(s)}\left(z^{\prime \prime}\right)\right)$ and non-intersecting $\cup \mathscr{D}^{(0)}$ such that either 
the distances from its end-points to $z^{\prime \prime}$ are equal to $C_{3} \varepsilon$ or only one of these distances is equal to $C_{3} \varepsilon$ and the other end-point belongs to $\bigcup_{\mathscr{D}(0)} \Gamma^{(s)}\left(\mathscr{D}^{(0)}\right)\left(\bigcup_{\mathscr{D}(0)} \Gamma^{(u)}\left(\mathscr{D}^{(0)}\right)\right)$;

2) for arbitrary $\delta^{(u)}\left(z_{1}^{\prime \prime}\right), \delta^{(u)}\left(z_{2}^{\prime \prime}\right), z_{1}^{\prime \prime}, z_{2}^{\prime \prime} \in Q_{\varepsilon}^{\prime \prime}$

$$
\min _{\substack{y_{1} \in \delta^{(u)}\left(z_{1}^{\prime \prime}\right) \\ y_{2} \in \delta(u)\left(z_{2}^{\prime \prime}\right)}} \operatorname{dist}\left(y_{1}, y_{2}\right) \geqq C_{5} \varepsilon
$$

From 1) it follows that $\delta^{(u)}\left(z^{\prime \prime}\right) \cap \bigcup_{|k| \leqq m} S_{k}=\emptyset, \delta^{(s)}\left(z^{\prime \prime}\right) \cap \bigcup_{|k| \leqq m} S_{k}=\emptyset$ for every $z^{\prime \prime} \in Q_{\varepsilon}^{\prime \prime}$.

Refraining for a moment from the proof of Lemma 3 we shall complete the construction of a partition $\xi_{0}$ which we need. We have increasing curves $\delta^{(u)}(z)$ constructed for

$$
z \in \bigcup_{k=0}^{m} \mathscr{P}_{\varepsilon}\left(S_{-k}\right) \cup \bigcup_{k=1}^{m} T^{-k} \mathscr{P}_{\varepsilon}\left(S_{0}\right)
$$

and all $z \in Q_{\varepsilon}^{\prime \prime}$. The first curves satisfy the relation $\delta^{(u)}(z) \cong T \delta_{1}^{(u)}\left(T^{-1} z\right)$ where $\delta_{1}^{(u)}\left(T^{-1} z\right)$ is a component of continuity containing $T^{-1} z$. In a similar way we have decreasing curves $\delta^{(s)}(z)$ for

$$
z \in \bigcup_{k=0}^{m} \mathscr{P}_{\varepsilon}\left(S_{k}\right) \cup \bigcup_{k=1}^{m} T^{k} \mathscr{P}_{\varepsilon}\left(S_{0}\right)
$$

and all $z \in Q_{\varepsilon}^{\prime \prime}$. The first curves satisfy the relation $\delta^{(s)}(z) \subseteq T^{-1} \delta_{1}^{(s)}(T z)$ where $\delta_{1}^{(s)}(T z)$ is a component of continuity containing $T z$. We can shorten or lengthen each constructed curve $\delta^{(u)}(z), \delta^{(s)}(z), z \in Q_{\varepsilon}^{\prime \prime}$ not more than $C_{6} \varepsilon$ in such a way that both relations will be valid and the end-points of each new segments $\delta^{(u)}(z), \delta^{(s)}(z)$ will belong to some $\delta^{(s)}\left(z^{\prime}\right), \delta^{(u)}\left(z^{\prime}\right)$ and the distances of each end-points of new $\delta^{(s)}\left(z^{\prime}\right)$, $\delta^{(u)}\left(z^{\prime}\right)$ to the end-points of new $\delta^{(u)}(z), \delta^{(s)}(z)$ are not less than $C_{6} \varepsilon$. The constant $C_{6}$ must be sufficiently small. Now we define $\xi_{0}$ as a partition the elements of which are connected open sets the boundaries of which are contained in

$$
\cup \delta^{(s)}(z) \cup \cup \delta^{(u)}(z) \cup \bigcup_{|k| \leqq m} S_{k} .
$$

Thus elements of each necklace $\mathfrak{N}\left(S_{k}\right),|k| \leqq m$ are elements of the partition $\xi_{0}$. It is worthwhile to mention that elements of the partition $\xi_{0}$ which are non-adjacent polygons are quadrilaterals.

Proof of Lemma 3. Let there be given two arbitrary increasing straight segments $\delta^{(u)}\left(z_{1}\right), \delta^{(u)}\left(z_{2}\right)$ the lengths of which are not more than $2 C_{3} \varepsilon$ and $C_{5}>0$ is sufficiently small. Suppose that $\delta^{(u)}\left(z_{i}\right) \cap \cup \mathscr{D}^{(0)}=\emptyset$ and $\operatorname{dist}\left(z, \delta^{(u)}\left(z_{2}\right)\right) \geqq C_{5} \varepsilon$ for some $z \in \delta^{(u)}\left(z_{1}\right)$. We shall show that if $m$ is large enough and $\varepsilon$ is small enough then $\operatorname{dist}\left(\delta^{(u)}\left(z_{1}\right), \delta^{(u)}\left(z_{2}\right)\right) \geqq \frac{1}{2} C_{5} \varepsilon$.

The curves of the set $\bigcup_{|k| \leqq m} S_{k}$ cut phase space $M_{1}$ on connected domains $F_{1}$, $F_{2}, \ldots, F_{r_{m}}$. Inside each domain the mappings $T^{m}$ and $T^{-m}$ are continuous. Let the $n$-th convergent of the continuous fraction $\kappa^{(u)}(z)$ be $P_{n}(z) / Q_{n}(z), b_{i}(z)$ are its elements. From the recurrent equations for $P_{n}(z), Q_{n}(z)$ we have

$$
Q_{n}-Q_{n-2}=b_{n} b_{n-1} Q_{n-2}+b_{n} / b_{n-2}\left(Q_{n-2}-Q_{n-4}\right), \quad n \geqq 5 .
$$


The elements $b_{i}$ are positive and bounded from below by the length of the least interval between two subsequent reflections ${ }^{4}$ and the least curvature of $\partial Q$ multiplied by 2 . Therefore the denominators $Q_{n}(z)$ are bounded from below by some constant independent of $z$. From the equality

$$
\frac{P_{n+1}}{Q_{n+1}}-\frac{P_{n}}{Q_{n}}=(-1)^{n} \frac{1}{Q_{n} Q_{n+1}}
$$

and from the fact that $\kappa^{(u)}(z)$ lies always between $P_{n}(z) / Q_{n}(z)$ and $P_{n+1}(z) / Q_{n+1}(z)$ we derive that for each $\alpha>0$ one can find $m=m(\alpha)$ such that for every $z_{1}, z_{2} \in \operatorname{Int} F_{i}$, $i=1,2, \ldots, r_{m}$

$$
\left|\kappa^{(u)}\left(z_{1}\right)-\kappa^{(u)}\left(z_{2}\right)\right|<\alpha, \quad\left|\kappa^{(s)}\left(z_{1}\right)-\kappa^{(s)}\left(z_{2}\right)\right|<\alpha .
$$

The assertion formulated above can easily be derived from these inequalities.

Let us take a component $\partial M_{1}^{(i)}$ and introduce coordinates $r, \varphi$ (see Sect. 1). There exists a number $C_{5}<1$ such that one can decompose $\partial M_{1}^{(i)}$ into rectangular cells with sides equal to $C_{5}^{2} \varepsilon . \partial M_{1}^{(i)}$ being a cylinder each cell can be described by two integers (numbers with respect to $r$ and $\varphi$ coordinate correspondingly). We can introduce a lexicographical ordering in the set of cells. Also we can assume that none of the points of $Q_{\varepsilon}^{\prime}$ belongs to a boundary of a cell. If $C_{5}$ is sufficiently small then each cell contains not more than one point of $Q_{\varepsilon}^{\prime}$. Therefore the ordering of the cells induces some ordering of the points of $Q_{\varepsilon}^{\prime}$. The set $\partial M_{1}^{(i)} \backslash \cup \mathscr{D}^{(0)}$ consists of a finite number of connected domains. If follows from the definition of the set $Q_{\varepsilon}^{\prime}$ that a cell intersecting $\cup \mathscr{D}^{(0)}$ contains no points of $Q_{\varepsilon}^{\prime}$ and such cells we shall not consider.

Suppose that for $z_{1}<z_{2}<\ldots<z_{k}, z_{i} \in Q_{\varepsilon}^{\prime}$ the shift is already performed and new curves are constructed. Let us take the next point $z_{k+1}>z_{k}$. Then $C_{5} \varepsilon^{-}$ neighbourhood on the line $\varphi=$ const of the point $z_{k+1}$ intersects with some curves $\delta^{(u)}\left(z_{i}^{\prime}\right)$ constructed earlier via the induction hypothesis. The total number of such curves is not more than $N$, where $N$ depends only on $C_{3}$. Therefore if we consider $2\left[C_{5}^{-1}\right]$ cells intersecting the line $\varphi=$ const containing $z_{k+1}^{\prime}$ for which the distances to $z_{k+1}^{\prime}$ does not exceed $C_{5} \varepsilon$ there exists a finite number of cells which intersect none of the curves $\delta^{(u)}\left(z_{i}^{\prime}\right)$. If we take $z_{k+1}^{\prime}$ equal to the center of one of these cells and construct an increasing straight segment passing through $z_{k+1}^{\prime}$ then due to the assertion stated above its distance from the curves of the same monotonicity constructed earlier is not less than $\frac{1}{2} C_{5}^{2} \varepsilon$. We can choose the cell and its center $z_{k+1}^{\prime}$ in such a way that the last assertion will be valid simultaneously for increasing and decreasing curves. Let the end of the increasing (decreasing) straight segment passing through $z_{k+1}^{\prime}$ belong to $\delta^{(s)} \subset \Gamma^{(s)}\left(\cup \mathscr{D}^{(0)}\right)\left[\delta^{(u)} \subset \Gamma^{(u)}\left(\cup \mathscr{D}^{(0)}\right)\right]$. It is obvious that we can take $z_{k+1}^{\prime}$ in such a way that the distance of this end to the nearest end of $\delta^{(s)}\left(\delta^{(u)}\right)$ is not less than $\frac{\gamma}{4} C_{3} \varepsilon$. Q.E.D.

4 It is true if $Q$ is a domain on two-dimensional torus. If $Q$ is a domain on Euclidean plane then the interval between two consecutive reflections is not bounded from below by a positive number. In this case one can make use of the fact that there exists a constant $L$ such that any point $q \in \Gamma_{\imath} \cap \Gamma_{j}$ has a neighbourhood $U_{q}$ with the property that any segment of the orbit completely containing in $U_{q}$ has no more than $L$ reflections from the boundary. Therefore in this case one must consider $T^{L}$ instead of $T$ 


\section{Construction of a pre-Markov Partition}

Let $\xi_{0}$ be the partition constructed in Sect. 3, $\Gamma^{(s)}\left(\xi_{0}\right)=\bigcup_{i} \delta_{i}^{(s)}\left(\xi_{0}\right), \Gamma^{(u)}\left(\xi_{0}\right)$ $=\bigcup_{i} \delta_{i}^{(u)}\left(\xi_{0}\right)$ where $\delta_{i}^{(s)}\left(\xi_{0}\right)\left(\delta_{i}^{(u)}\left(\xi_{0}\right)\right)$ are maximal decreasing (increasing) connected components of the boundary. Some of $\delta_{i}^{(s)}\left(\xi_{0}\right), \delta_{i}^{(u)}\left(\xi_{0}\right)$ consist of several smooth connected components. It is important to remark that if $\delta_{i}^{(s)}\left(\xi_{0}\right)\left(\delta_{i}^{(u)}\left(\xi_{0}\right)\right)$ consists of several smooth components than there exists $\delta_{j}^{(s)}\left(\xi_{0}\right)\left(\delta_{j}^{(u)}\left(\xi_{0}\right)\right)$ which has only one smooth component and for some $k, 0<k \leqq 2 m$,

$$
T^{-k} \delta_{j}^{(s)}\left(\xi_{0}\right) \cup T^{-k-1} \delta_{j}^{(s)}\left(\xi_{0}\right) \supseteqq \delta_{i}^{(s)}\left(\xi_{0}\right) \quad\left[T^{k} \delta_{j}^{(u)}\left(\xi_{0}\right) \cup T^{k+1} \delta_{j}^{(u)}\left(\xi_{0}\right) \supseteqq \delta_{i}^{(u)}\left(\xi_{0}\right)\right] .
$$

In what follows we shall denote $\delta_{i_{1}}^{(s)}\left(\xi_{0}\right), \delta_{i_{2}}^{(u)}\left(\xi_{0}\right)$ consisting of one smooth component by tilde: $\tilde{\delta}_{i_{1}}^{(s)}\left(\xi_{0}\right), \tilde{\delta}_{i_{2}}^{(u)}\left(\xi_{0}\right)$. The construction described in Sect. 3 permits in fact to get a partition $\xi_{0}$ if only components of the boundaries $\tilde{\delta}_{i_{1}}^{(s)}\left(\xi_{0}\right), \tilde{\delta}_{i_{2}}^{(u)}\left(\xi_{0}\right)$ are given. Certainly we assume that the constants $C_{1}, C_{2}, C_{3}, \ldots$ are fixed too and satisfy the relations mentioned in Sect. 3 . It should also be noted that a partition $\xi_{0}$ is certainly non-unique. However if the partition $\xi_{0}$ is chosen and we change slightly (not more than on $C_{5} \varepsilon$ ) all $\tilde{\delta}_{i_{1}}^{(s)}\left(\xi_{0}\right), \tilde{\delta}_{i_{2}}^{(u)}\left(\xi_{0}\right)$ then we can construct other components of the boundary and the corresponding partition uniquely.

Theorem 1. Let $\varepsilon$ be sufficiently small and $m$ sufficiently large. For each set of $\tilde{\delta}_{i_{1}}^{(s)}\left(\xi_{0}\right), \tilde{\delta}_{i_{2}}^{(u)}\left(\xi_{0}\right)$ one can find new decreasing and increasing curves $\tilde{\delta}_{i_{1}}^{(s)}, \tilde{\delta}_{i_{2}}^{(u)}$ in such a way that

1. $\operatorname{dist}\left(\tilde{\delta}_{i_{1}}^{(s)}, \tilde{\delta}_{i_{1}}^{(s)}\left(\xi_{0}\right)\right) \leqq C_{7} \Lambda_{\min }^{-m}$

2. if $\tilde{\delta}_{i_{1}}^{(s)}\left(\xi_{0}\right) \cap \tilde{\delta}_{i_{2}}^{(u)}\left(\xi_{0}\right) \neq \emptyset$, then $\tilde{\delta}_{i_{1}}^{(s)} \cap \tilde{\delta}_{i_{2}}^{(u)} \neq \emptyset$;

3. if using $\tilde{\delta}_{i_{1}}^{(s)}, \delta_{i_{2}}^{(u)}$ we construct other components and the partition $\eta_{0}$ as in Sect. 3 then

$$
T^{m} \Gamma^{(s)}\left(\eta_{0}\right) \subseteq \Gamma^{(s)}\left(\eta_{0}\right), \quad T^{-m} \Gamma^{(u)}\left(\eta_{0}\right) \subseteq \Gamma^{(u)}\left(\eta_{0}\right) .
$$

Proof of the theorem proceeds the same way as the proof of the similar theorem in $[2,3]$. Let us take an arbitrary component $\tilde{\delta}_{i_{1}}^{(u)}\left(\xi_{0}\right)$. By construction the mapping $T^{-m}$ is continuous on $\tilde{\delta}_{i_{1}}^{(u)}\left(\xi_{0}\right)$. Moreover $T^{-m} \tilde{\delta}_{i_{1}}^{(u)}\left(\xi_{0}\right)$ is also an increasing curve. Indeed, there exists at least one point $z_{0} \in \tilde{\delta}_{i_{1}}^{(u)}\left(\xi_{0}\right)$ where the line tangent to $T^{-m} \delta_{i_{1}}^{(u)}\left(\xi_{0}\right)$ at $T^{-m} z_{0}$ is at an angle $\varphi^{(u)}\left(T^{-m} z_{0}\right)$. The final conclusion follows from the smallness of $\varepsilon$.

Let the end-points of $\tilde{\delta}_{i_{1}}^{(u)}\left(\xi_{0}\right)$ belong to $\delta_{j_{1}}^{(s)}\left(\xi_{0}\right), \delta_{j_{2}}^{(s)}\left(\xi_{0}\right)$. Certainly it may happen that $\delta_{j_{1}}^{(s)}\left(\xi_{0}\right), \delta_{j_{2}}^{(s)}\left(\xi_{0}\right)$ have more than one smooth component. We consider $T^{-m} \delta_{j_{1}}^{(s)}\left(\xi_{0}\right), T^{-m} \delta_{j_{2}}^{(s)}\left(\xi_{0}\right)$. There can appear the following possibilities.

$\mathrm{a}_{1}$. Both $\delta_{j_{1}}^{(s)}\left(\xi_{0}\right), \delta_{j_{2}}^{(s)}\left(\xi_{0}\right)$ consist of a single smooth component. The lengths of $\delta_{j_{1}}^{(s)}\left(\xi_{0}\right), \delta_{j_{2}}^{(s)}\left(\xi_{0}\right)$ are not less than $\frac{\gamma}{2} C_{3} \varepsilon$. Therefore the lengths of $T^{-m} \delta_{j_{1}}^{(s)}\left(\xi_{0}\right)$, $T^{-m} \delta_{j_{2}}^{(s)}\left(\xi_{0}\right)$ are not less $\Lambda_{\min }^{m} \frac{C_{3} \varepsilon \gamma}{2}$. We can find $\tilde{\delta}_{i_{2}}^{(u)}\left(\xi_{0}\right)$ such that

1) $\operatorname{dist}\left(x, \tilde{\delta}_{i_{2}}^{(u)}\left(\xi_{0}\right)\right) \leqq \frac{1}{4} C_{3} \varepsilon \gamma$ for every point $\underset{\tilde{\delta} \in T^{-m}}{-m} \tilde{\delta}_{i_{1}}^{(u)}\left(\xi_{0}\right)$;

2) both $T^{-m} \delta_{j_{1}}^{(s)}\left(\xi_{0}\right), T^{-m} \delta_{j_{2}}^{(s)}\left(\xi_{0}\right)$ intersect $\tilde{\delta}_{j_{2}}^{(u)}\left(\xi_{0}\right)$ and the distance between the points of intersection and the end-points of $\tilde{\delta}_{i_{2}}^{(u)}\left(\xi_{0}\right)$ is not less than $\frac{1}{4} C_{3} \varepsilon \gamma$.

If $\delta^{(u)} \subset \tilde{\delta}_{i_{2}}^{(u)}\left(\xi_{0}\right)$ is the segment bounded by the points of intersection we take $T^{m} \delta^{(u)}=\tilde{\delta}_{i_{1}}^{(u)}$. 
Fig. 12

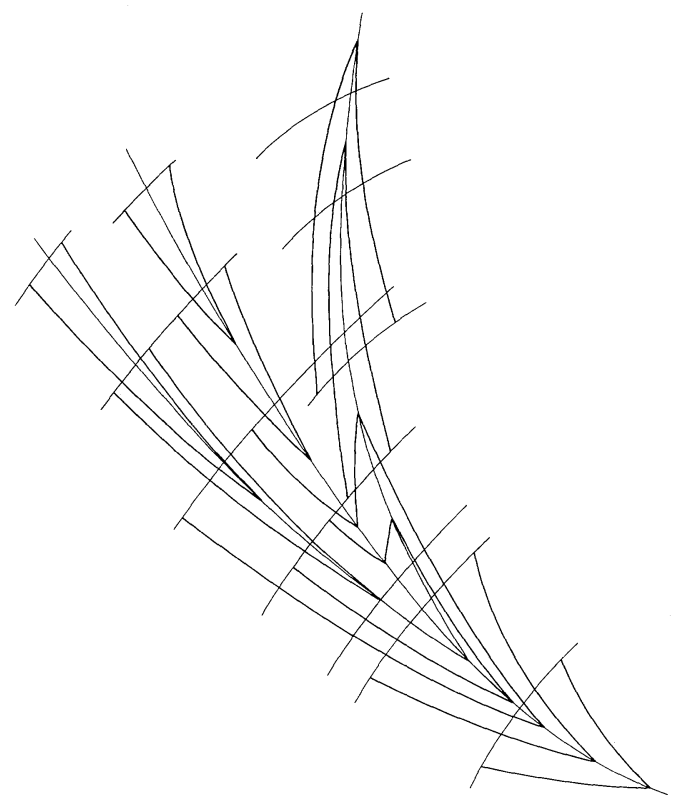

$\mathrm{a}_{2}$. One of $\delta_{j_{1}}^{(s)}\left(\xi_{0}\right), \delta_{j_{2}}^{(s)}\left(\xi_{0}\right)$ has at least two smooth components. Let $\delta_{j_{1}}^{(s)}\left(\xi_{0}\right)$ have this property. In this case we choose $\delta^{(u)}$ in such a way that the property 1 ) is valid. If $z_{1}=\delta_{i_{1}}^{(u)}\left(\xi_{0}\right) \cap \delta_{j_{1}}^{(s)}\left(\xi_{0}\right)$ then the end-point of $\delta^{(u)}$ corresponding to $T^{-m} z_{1}$ lies on the same smooth component of $T^{-m} \delta_{j_{1}}^{(s)}\left(\xi_{0}\right)$ as $T^{-m} z_{1}$.

Now we have a new system of curves $\tilde{\delta}_{i_{1}}^{(u)}$ with the end-points belonging to the same smooth components of $\Gamma^{(s)}\left(\xi_{0}\right)$ as $\tilde{\delta}_{i_{1}}^{(u)}\left(\xi_{0}\right)$. It may happen that some endpoints of the components of $\Gamma^{(s)}\left(\xi_{0}\right)$ don't lie on $\bigcup_{i_{1}} \tilde{\delta}_{i_{1}}^{(u)}$.

Changing $T^{-m}$ to $T^{m}$ and vice versa we get in the same way a new system of curves $\tilde{\delta}_{i_{1}}^{(s)}$. We can choose them in such a way that the end-points of each $\tilde{\delta}_{i_{1}}^{(s)}$ lie on some $\tilde{\delta}_{i_{2}}^{(u)}$ and their distances to the end-points of $\tilde{\delta}_{i_{2}}^{(u)}$ are not less than $\frac{C_{3}}{4} \varepsilon \gamma$. Next we can choose new $\tilde{\tilde{\delta}}_{i_{1}}^{(u)}$ in such a way that $T^{-m} \tilde{\tilde{\delta}}_{i_{1}}^{(u)} \subset \tilde{\delta}_{i_{2}}^{(u)}\left(\xi_{0}\right)$ and the end-points of $\tilde{\tilde{\delta}}_{i_{1}}^{(u)}$ belong to $\bigcup_{j} \tilde{\delta}_{j}^{(s)}$.

Now we denote $\tilde{\delta}_{i_{1}}^{(u)}\left(\xi_{1}\right)=\tilde{\tilde{\delta}}_{i_{1}}^{(u)}, \tilde{\delta}_{i_{2}}^{(s)}\left(\xi_{1}\right)=\tilde{\delta}_{i_{2}}^{(s)}$ and using them construct a new partition which we denote $\xi_{1}$. We evidently have

$$
\begin{gathered}
T^{-m} \Gamma^{(u)}\left(\xi_{1}\right) \cong \Gamma^{(u)}\left(\xi_{1}\right) \cup \Gamma^{(u)}\left(\xi_{0}\right), \\
T^{m} \Gamma^{(s)}\left(\xi_{1}\right) \cong \Gamma^{(s)}\left(\xi_{1}\right) \cup \Gamma^{(s)}\left(\xi_{0}\right) .
\end{gathered}
$$

Suppose now that we have already constructed a sequence of partitions $\xi_{0}, \xi_{1}, \ldots, \xi_{n}$ with the same properties as $\xi_{0}$ and

$$
T^{-m} \Gamma^{(u)}\left(\xi_{n}\right) \subseteq \Gamma^{(u)}\left(\xi_{n}\right) \cup \Gamma^{(u)}\left(\xi_{n-1}\right), \quad T^{m} \Gamma^{(s)}\left(\xi_{n}\right) \subset \Gamma^{(s)}\left(\xi_{n}\right) \cup \Gamma^{(s)}\left(\xi_{n-1}\right) .
$$


The same arguments as above give the possibility to construct $\xi_{n+1}$, for which

$$
\begin{gathered}
T^{-m} \Gamma^{(u)}\left(\xi_{n+1}\right) \subseteq \Gamma^{(u)}\left(\xi_{n+1}\right) \cup \Gamma^{(u)}\left(\xi_{n}\right), \\
T^{m} \Gamma^{(s)}\left(\xi_{n+1}\right) \cong \Gamma^{(s)}\left(\xi_{n+1}\right) \cup \Gamma^{(s)}\left(\xi_{n}\right) .
\end{gathered}
$$

It is easy to see that $\operatorname{dist}\left(\Gamma^{(u)}\left(\xi_{n+1}\right), \Gamma^{(u)}\left(\xi_{n}\right)\right) \leqq \operatorname{const} \Lambda_{\min }^{-m n}, \operatorname{dist}\left(\Gamma^{(s)}\left(\xi_{n+1}\right), \Gamma^{(s)}\left(\xi_{n}\right)\right)$ $\leqq$ const $\Lambda_{\min }^{-m n}$. Therefore the limit $\lim _{n \rightarrow \infty} \xi_{n}=\eta_{0}$ exists and satisfies all the conditions of the Theorem 1. Q.E.D.

Partition $\eta_{0}$ is not a Markov partition because its elements are not parallelograms. It is easy to see that the elements $C\left(\eta_{0}\right)$ of $\eta_{0}$ are curvilinear polygons for which the total number of sides does not exceed five (see Fig. 12).

If the boundary of $C\left(\eta_{0}\right)$ does not intersect $\bigcup_{|k| \leqq m} T^{k} S_{0}$ then $C\left(\eta_{0}\right)$ is a quadrilateral. The elements $C\left(\eta_{0}\right)$ for which $\partial\left(C\left(\eta_{0}\right)\right) \cap S_{k} \neq \emptyset$ will be called adjacent polygons. Certainly in the last expression we must take $|k| \leqq m$ but later we shall use this term in a more general situation. We show in the next section how to pass from the partition $\eta_{0}$ to a Markov partition. The partition constructed in Theorem 1 will be called a pre-Markov partition.

\section{Transition from a pre-Markov Partition to a Markov Partition}

In this section we prove the following

Theorem. Let there be given a finite partition $\eta_{0}$ of the phase space $M_{1}$ such that

1) each element $C_{i}$ of $\eta_{0}$ is a polygon which is either adjacent or non-adjacent; all non-adjacent polygons are quadrilaterals and adjacent polygons are triangles, quadrilaterals or pentagons;

2) $\tilde{\Gamma}^{(s)}\left(\eta_{0}\right) \supset \bigcup_{k=1}^{m} S_{-k} ; \tilde{\Gamma}^{(u)}\left(\eta_{0}\right) \supset \bigcup_{k=1}^{m} S_{k}$;

3) $T^{m} \Gamma^{(s)}\left(\eta_{0}\right) \cong \Gamma^{(s)}\left(\eta_{0}\right) ; T^{-m} \Gamma^{(u)}\left(\eta_{0}\right) \subseteq \Gamma^{(u)}\left(\eta_{0}\right)$.

Then there exists a countable partition $\eta, \eta \geqq \eta_{0}$, such that

$a_{1}$ ) each element of $\eta$ is a parallelogram;

$\mathrm{a}_{2}$ ) $\eta$ is a Markov partition with respect to $T^{m}$, i.e.

$$
T^{m} \gamma_{\eta}^{(s)}(x) \subset \gamma_{\eta}^{(s)}\left(T^{m} x\right), T^{-m} \gamma_{\eta}^{(u)}(x) \cong \gamma_{\eta}^{(u)}\left(T^{-m} x\right)
$$

for almost all $x$.

Proof of the theorem is given below. The property 3) was called the preMarkov property of a partition. The partition $\eta_{0}$ constructed in Sect. 4 satisfies conditions 1)-3). We shall use again the notation $T_{1}=T^{m}$.

The whole construction will have an inductive character. We put $\eta_{0}^{+}=\eta_{0}$ and suppose that partitions $\eta_{0}^{+} \leqq \eta_{1}^{+} \leqq \ldots \leqq \eta_{n}^{+}$are already constructed which have the following properties :

1) there exists a closed neighbourhood $\mathscr{D}_{n}^{(m)}$ of $\bigcup_{|k| \leqq m} S_{k}$ consisting of elements $C_{\eta_{n}^{+}}$of $\eta_{n}^{+}$such that $\eta_{n}^{+}\left|\mathscr{D}_{n}^{(m)}=\bigvee_{|k| \leqq n} T_{1}^{k} \eta_{0}\right| \mathscr{D}_{n}^{(m)}$ and every element $C_{\eta_{n}^{+}} \subset \mathscr{D}_{n}^{(m)}$ is a 
polygon; the set $\mathscr{D}_{n}^{(m)}$ is called the $n$-th necklace of $\bigcup_{|k| \leqq m} S_{k}$;

2) $\mathscr{D}_{n}^{(m)} \cong \mathscr{D}_{n-1}^{(m)} \cong \ldots \subseteq \mathscr{D}_{0}^{(m)}$.

For every $l, l \leqq n$, the set $\bigcup_{k=0}^{l} T_{1}^{k} \mathscr{D}_{n}^{(m)}=\mathscr{D}_{n}^{(l m)}$ is called the $n$-th necklace of $\bigcup_{-m \leqq k \leqq l m} S_{k}$.

We begin the construction of $\eta_{n+1}^{+}$with the definition of $\mathscr{D}_{n+1}^{(m)}$. For every $C_{\eta_{n}^{+}} \subset \mathscr{D}_{n}^{(m)}$ we consider $\bigvee_{|k| \leqq n+1} T_{1}^{k} \eta_{0} \mid C_{\eta_{n}^{+}}$.

If $K$ is the total number of the elements of $\eta_{0}$ then $\bigvee_{|k| \leqq n+1} T_{1}^{k} \eta_{0} \mid C_{\eta_{n}^{+}}$consists of not more than $K^{2}$ elements. It is easy to see that each of them is a connected set and a polygon. The set of those elements of $\bigvee_{|k| \leqq n+1} T_{1}^{k} \eta_{0} \mid \mathscr{D}_{m}^{(n)}$ which are adjacent to $\bigcup_{|k| \leqq m} S_{k}$ is by definition the $(n+1)$-th necklace of $\bigcup_{|k| \leqq m} S_{k}$, their union being denoted by $\mathscr{D}_{n+1}^{(m)}$. We also put

$$
\eta_{n+1}^{+}\left|\mathscr{D}_{n+1}^{(m)}=\underset{|k| \leqq n+1}{\bigvee} T_{1}^{k} \eta_{0}\right| \mathscr{D}_{n+1}^{(m)} .
$$

Next we define for $1 \leqq l \leqq n+1$

$\left.\eta_{n+1}^{+} \mid\left(T_{1}^{l} \mathscr{D}_{n+1}^{(m)}-\quad \bigcup_{0 \leqq k<l} T_{1}^{k} \mathscr{D}_{n+1}^{(m)}\right)={ }_{-n+l-2} \bigvee_{<\leqq n+1+l} T_{1}^{k} \eta_{0}\right)\left(T_{1}^{l} \mathscr{D}_{n+1}^{(m)}-\bigcup_{0 \leqq k<l}\right.$ $\left.T_{1}^{k} \mathscr{D}_{n+1}^{(m)}\right)$

and

$$
\eta_{n+1}^{+}\left|\left(M_{1}-\bigcup_{l=0}^{n+1} T_{1}^{l} \mathscr{D}_{n+1}^{(m)}\right)=\eta_{n}^{+}\right|\left(M_{1}-\bigcup_{l=0}^{n+1} T_{1}^{l} \mathscr{D}_{n+1}^{(m)}\right) .
$$

Thus $\eta_{n+1}^{+}$is completely defined.

We shall investigate in more detail properties of the sequence $\left\{\eta_{n}^{+}\right\}$. It is easy to see that $\mathscr{D}_{n}^{(m)}$ is contained in the (const $\Lambda_{\min }^{-n \cdot m} \cdot \varepsilon$ )-neighbourhood of $\bigcup_{|k| \leq m} S_{k}$. Therefore $v\left(\mathscr{D}_{n}^{(m)}\right) \leqq$ const $\Lambda_{\min }^{-m n} \cdot \varepsilon$ and

$$
v\left(\bigcup_{0 \leqq k \leqq n+1} T_{1}^{k} \mathscr{D}_{n}^{(m)}\right) \leqq \operatorname{const}(n+1) \Lambda_{\min }^{-m \cdot n} \cdot \varepsilon .
$$

Lemma 5.1. $T_{1} \Gamma^{(s)}\left(\eta_{n}^{+}\right) \subset \Gamma^{(s)}\left(\eta_{n}^{+}\right), n=0,1, \ldots$

Proof. For $n=0$ the statement of the lemma follows directly from the pre-Markov property of $\eta_{0}=\eta_{0}^{+}$. We assume that it is already proven for $\eta_{0}^{+} \leqq \eta_{1}^{+} \leqq \ldots \leqq \eta_{n-1}^{+}$ and we shall prove it for $\eta_{n}^{+}$.

Let $\Gamma_{\left.\mathscr{O} \tilde{h}^{n}\right)}^{(\mathrm{s})}\left(\eta_{n}^{+}\right)$be the union of decreasing boundaries of all polygons belonging to $\mathscr{D}_{n}^{(m)}$. It follows easily from the construction of $\eta_{n}^{+}$that $\Gamma_{\mathscr{D}\left(\begin{array}{c}n \\ n\end{array}\right)}^{(s)}\left(\eta_{n}^{+}\right) \subset \Gamma^{(s)}\left(\bigvee_{|k| \leqq n} T_{1}^{k} \eta_{0}\right)$. We have also

$$
\Gamma^{(s)}\left(\eta_{n}^{+}\right)=\Gamma^{(s)}\left(\eta_{n-1}^{+}\right) \cup\left(\bigcup_{0 \leqq k \leqq n} T_{1}^{k} \Gamma_{\left.\mathscr{D}_{n}\right)}^{(s)}\left(\eta_{n}^{+}\right)\right) .
$$


In view of our inductive assumption $T_{1} \Gamma^{(s)}\left(\eta_{n-1}^{+}\right) \subset \Gamma^{(s)}\left(\eta_{n-1}^{+}\right)$. Therefore

$$
T_{1}\left(\Gamma^{(s)}\left(\eta_{n}^{+}\right)\right)=T_{1}\left(\Gamma^{(s)}\left(\eta_{n-1}^{+}\right)\right) \cup \bigcup_{1 \leqq k \leqq n} T_{1}^{k} \Gamma_{\mathscr{D}_{n}^{(m)}}^{(s)}\left(\eta_{n}^{+}\right) \cup T_{1}^{n+1} \Gamma_{\mathscr{D}_{n}^{(m)}}^{(s)}\left(\eta_{n}^{+}\right)
$$

From the pre-Markov property of $\eta_{0}$ we have

$$
T_{1}^{n+1} \Gamma_{\mathscr{D}_{n}^{(m)}}^{(s)}\left(\eta_{n}^{+}\right) \subset T_{1} \Gamma^{(s)}\left(\eta_{0}\right) \subset \Gamma^{(s)}\left(\eta_{0}\right) \subset \Gamma^{(s)}\left(\eta_{n}^{+}\right)
$$

because $\eta_{0} \leqq \eta_{n}^{+}$. Now from $(*),(* *),(* * *)$ we get the desired result. Q.E.D.

Lemma 5.2. The connected components of elements $C\left(\eta_{n}^{+}\right)$which are polygons, nonadjacent to the set $\bigcup_{-m \leqq k \leqq m n} S_{k}$ are quadrilaterals.

Proof. It follows easily from the construction of $\eta_{n}^{+}$that each connected component of $C\left(\eta_{n}^{+}\right)$satisfying the assumption of Lemma 5.2 is a polygon whose boundary consists of a finite number of increasing and decreasing curves.

We shall show that two components of the boundary of the same monotonicity cannot intersect. Let us assume that this is not true and there exists a point $x \in \delta_{1}^{(s)} \cap \delta_{2}^{(s)}$ where $\delta_{1}^{(s)}, \delta_{2}^{(s)}$ are two decreasing curves which are parts of the boundary of $C\left(\eta_{n}^{+}\right)$. We have the inclusion $\Gamma^{(s)}\left(\eta_{n}^{+}\right) \cong \Gamma^{(s)}\left(T_{1}^{-n} \eta_{0}\right)$ which is an immediate consequence of the construction. Therefore from Lemma 5.1

$$
T_{1}^{n} x=T_{1}^{n}\left(\delta_{1}^{(s)} \cap \delta_{2}^{(s)}\right)=T_{1}^{n} \delta_{1}^{(s)} \cap T_{1}^{n} \delta_{2}^{(s)} \subseteq \Gamma^{(s)}\left(\eta_{0}\right)
$$

which shows that $\Gamma^{(s)}\left(\eta_{0}\right)$ must have two intersecting components of the same monotonicity. But this is obviously wrong. Thus we have that the number of smooth components of $\partial\left(C\left(\eta_{n}^{+}\right)\right)$is necessarily even. Similar arguments can be applied to $\delta_{1}^{(u)} \cap \delta_{2}^{(u)}$ in view of inclusion $\Gamma^{(u)}\left(\eta_{n}^{+}\right) \subset \Gamma^{(u)}\left(T_{1}^{2 n} \eta_{0}\right)$.

Our considerations will have again an inductive character. Assume that the statement of the lemma is already established for all $\eta_{0}^{+} \leqq \eta_{1}^{+} \leqq \ldots \leqq \eta_{n}^{+}$. We shall show that it is valid also for $\eta_{n+1}^{+}$. We must consider only two cases.

1. Int $C\left(\eta_{n}^{+}\right) \cap \operatorname{Int} T_{1}^{n+1} \mathscr{D}_{n+1}^{(m)} \neq \emptyset$ and $C\left(\eta_{n}^{+}\right)$is non-adjacent to $\bigcup_{-m \leqq k \leqq m n} S_{k}$.

We shall show that the common boundary of the sets $\bigcup_{m n<l \leqq m(n+1)} T^{l} \mathscr{D}_{n+1}^{(m)}$, $C\left(\eta_{n}^{+}\right)-\bigcup_{m n<l \leqq m(n+1)} T^{l} \mathscr{D}_{n+1}^{(m)}$ is a union of smooth increasing curves whose endpoints belong to $\Gamma^{(s)}\left(C\left(\eta_{n}^{+}\right)\right)$. Suppose that this is wrong and a component of the boundary consists of several smooth components. None of these components can be a decreasing curve because it contradicts the pre-Markov property of $\eta_{n+1}^{+}$.

This means that all the components are increasing curves. But this is also impossible in view of the arguments given in the beginning of this proof. As a result we get that the common boundary of the sets $C\left(\eta_{n}^{+}\right)-\bigcup_{m n \leqq l \leqq m(n+1)} T^{l} \mathscr{D}_{n+1}^{(m)}$, $\bigcup_{m n \leqq l \leqq m(n+1)} T^{l} \mathscr{D}_{n+1}^{(m)}$ consists of increasing curves. The end-points of these curves belong to $\Gamma^{(s)}\left(C\left(\eta_{n}^{+}\right)\right)$because of pre-Markov property of $\eta_{n}^{+}$. Thus we see that $C\left(\eta_{n}^{+}\right) \cap \bigcup_{m n \leqq l \leqq m(n+1)} T^{l} \mathscr{D}_{n+1}^{(m)}$ is a finite number of strips and connected components of $C\left(\eta_{n}^{+}\right)-\bigcup_{m n \leqq l \leqq m(n+1)} T^{l} \mathscr{D}_{n+1}^{(m)}$ are quadrilaterals. 
2. $C\left(\eta_{n}^{+}\right) \subseteq \bigcup_{l=0}^{n-1} T_{1}^{l}\left(\mathscr{D}_{n-1}^{(m)}-\mathscr{D}_{n}^{(m)}\right)$ is a non-adjacent element of $\eta_{n}^{+}$.

The construction of $\eta_{n}^{+}$shows that it is sufficient to consider only non-adjacent elements belonging to $\mathscr{D}_{n-1}^{(m)}-\mathscr{D}_{n}^{(m)}$. We can represent such $C\left(\eta_{n}^{+}\right) \subset \mathscr{D}_{n}^{(m)}$ in the form $C\left(\eta_{n}^{+}\right)=T_{1}^{-1} C_{1} \cap \mathscr{D} \cap T_{1} C_{2}$ where $C_{1}, C_{2}, D$ are elements of $\bigvee_{|k| \leqq n-1} T_{1}^{k} \eta_{0}$ and $\mathscr{D} \subset \mathscr{D}_{n-1}^{(m)}$ is an element of $\eta_{n-1}^{+}$. We also point out that the partition $\bigvee_{|k| \leqq n-1} T_{1}^{k} \eta_{0}$ has the pre-Markov property and $C\left(\eta_{n}^{+}\right)$is a polygon whose boundary has an even number of smooth components. The last statement has in fact been proven earlier.

Assume now that $A=\mathscr{D} \cap T_{1} C_{2}$ is a polygon which is non-adjacent to the set $\bigcup_{-m \leqq k \leqq m n} S_{k}$. We shall show that it is a quadrilateral. Let us consider the polygon $T_{1} C_{2}$. Some components of its increasing boundary can belong to $\bigcup_{0<k \leqq m n} S_{k}$. But the increasing components whose parts belong also to the boundary of $A$ differ from them because $A$ in non-adjacent. We shall now show that parts of at least two increasing components of $T_{1} C_{2}$ are smooth components of the boundary of $A$. If none of them has this property then it means that the increasing part of $\partial A$ lies inside $T_{1} C_{2}$. But it contradicts the pre-Markov property of $\bigvee_{|k| \leqq n} T_{1}^{k} \eta_{0}$. If only one of them has this property then again we have a contradiction to the pre-Markov property of $\bigvee_{|k| \leqq n} T_{1}^{k} \eta_{0}$ because the total number of increasing components of the boundary of $A$ is not less than two and therefore at least one of them lies inside $T_{1} C_{2}$.

Thus we have at least two increasing components of the boundary of $T_{1} C_{2}$ intersecting $\mathscr{D}$. If $A$ is not a quadrilateral then at least two increasing components of the boundary of $A$ are also increasing components of $\mathscr{D}$. But in view of the preMarkov property of $\bigvee_{|k| \leqq n} T_{1}^{k} \eta_{0}$ it is possible only in case $\mathscr{D}=T_{1} C_{2}$ because otherwise increasing components of $A$ will lie inside $T_{1} C_{2}$.

If $\mathscr{D}=T_{1} C_{2}$ then $\mathscr{D}$ is a polygon which is non-adjacent to $\bigcup_{-m \leqq k \leqq m n} S_{k}$. This means that $\mathscr{D}$ cannot belong to the $n$-th necklace of $\bigcup_{|k| \leqq m} S_{k}$. As a result we get that $A$ has only two increasing components and therefore it is a quadrilateral.

The intersection $T_{1}^{-1} C_{1} \cap A$ can be treated in the same way. If $A=\mathscr{D} \cap T_{1} C_{2}$ is not a non-adjacent polygon then the same arguments applied to $T_{1}^{-1} C_{1} \cap \mathscr{D} \cap T_{1} C_{2}$ will yield the desired result. Q.E.D.

Taking everywhere $T_{1}^{-1}$ instead of $T_{1}$ we can construct in the same way the partitions $\eta_{0}=\eta_{0}^{-} \leqq \eta_{1}^{-} \leqq \ldots \leqq \eta_{n}^{-}$for which the same lemmas are valid.

Lemma 5.1'. $T_{1}^{-1} \Gamma^{(u)}\left(\eta_{n}^{-}\right) \subset \Gamma^{(u)}\left(\eta_{n}^{-}\right), n=0,1, \ldots$

Lemma 5.2'. Connected components of elements $C\left(\eta_{n}^{-}\right)$which are polygons, nonadjacent to $\bigcup_{-m n \leqq k \leqq m} S_{k}$ are quadrilaterals.

We also point out that the necklaces $\mathscr{D}_{n}^{(m)}$ coincide for both sequences of partitions. We shall introduce now the notion of rank for non-adjacent elements of 
the partitions $\eta_{n}^{+}$. If $C\left(\eta_{0}^{+}\right)$is a non-adjacent element of $\eta_{0}^{+}$then by definition its rank is equal to zero. Elements of $\eta_{1}^{+}$belonging to $\mathscr{D}_{0}^{(m)}-\mathscr{D}_{1}^{(m)}$ have a rank equal to one. Other non-adjacent elements of $\eta_{1}^{+}$have a rank equal to the rank of the containing element of $\eta_{0}^{+}$. In the general case we put the rank of an element of $\eta_{n}^{+}$ belonging to $\mathscr{D}_{n-1}^{(m)}-\mathscr{D}_{n}^{(m)}$ equal to $n$. The rank of an element of $\eta_{n}^{+}$belonging to $\left(T_{1}^{l} \mathscr{D}_{n-1}^{(m)}-\bigcup_{k=1}^{l-1} T_{1}^{k} \mathscr{D}_{n-1}^{(m)}\right)-\left(T_{1}^{l} \mathscr{D}_{n}^{(m)}-\bigcup_{k=1}^{l-1} T_{1}^{k} \mathscr{D}_{n}^{(m)}\right)$ is equal by definition to $n-l$, $1 \leqq l \leqq n$. By definition the rank of every non-adjacent element of $\eta_{n}^{+}$which is contained in a non-adjacent element of $\eta_{n-1}^{+}$is equal to the rank of this element of $\eta_{n-1}^{+}$.

In the same way we can define ranks of elements of $\eta_{n}^{-}$. Let us put $\eta_{n}=\eta_{n}^{+} \vee \eta_{n}^{-}$. If $C\left(\eta_{n}\right)=C_{1}\left(\eta_{n}^{+}\right) \cap C_{2}\left(\eta_{n}^{-}\right)$then we put $r_{+}\left(C\left(\eta_{n}\right)\right), r_{-}\left(C\left(\eta_{n}\right)\right)$ to be equal to the ranks of $C_{1}\left(\eta_{n}^{+}\right), C_{2}\left(\eta_{n}^{-}\right)$correspondingly. It is easy to see that $\eta_{n}$ has the preMarkov property.

Lemma 5.3. Let $C\left(\eta_{n}\right)=C_{1}\left(\eta_{n}^{+}\right) \cap C_{2}\left(\eta_{n}^{-}\right)$be such that $C_{1}\left(\eta_{n}^{+}\right)$and $C_{2}\left(\eta_{n}^{-}\right)$are nonadjacent elements of $\eta_{n}^{+}, \eta_{n}^{-}$correspondingly. If $r_{+}\left(C\left(\eta_{n}\right)\right)=k, r_{-}\left(C\left(\eta_{n}\right)\right)=l$ then 1$)$ $\Gamma^{(s)}\left(T_{1} C\left(\eta_{n}\right)\right) \subseteq \Gamma^{(s)}\left(C^{\prime}\left(\eta_{n-1}\right)\right)$ where either $r_{+}\left(C^{\prime}\left(\eta_{n-1}\right)\right)=k-1$ or $C^{\prime}\left(\eta_{n-1}\right)$ is polygon adjacent to $\bigcup_{m(n-k-1) \leqq p \leqq m(n-k)} S_{p}$. 2) $\Gamma^{(u)}\left(T_{1}^{-1} C\left(\eta_{n}\right)\right) \subset \Gamma^{(u)}\left(C^{\prime \prime}\left(\eta_{n-1}\right)\right)$ where either $r_{-}\left(C^{\prime \prime}\left(\eta_{n-1}\right)\right)=l-1$ or $C^{\prime \prime}\left(\eta_{n-1}\right)$ is a polygon adjacent to $\bigcup_{m(n-k-1) \leqq p \leqq m(n-k)} S_{-p}$.

The proof follows directly from the definitions.

Corollary. In conditions of Lemma 5.3

$$
\Gamma^{(s)}\left(T_{1}^{k} C\left(\eta_{n}\right)\right) \subset \Gamma^{(s)}\left(C_{1}\left(\eta_{0}\right)\right), \quad \Gamma^{(u)}\left(T_{1}^{-l} C\left(\eta_{n}\right)\right) \subset \Gamma^{(u)}\left(C_{2}\left(\eta_{0}\right)\right) .
$$

Let $\eta=\bigvee_{n} \eta_{n}$. We shall show that $\eta$ is a Markov partition whose elements are parallelograms and satisfies all assertions of theorem.

First we remark that it follows easily from the construction that each element $C(\eta)$ has \pm -ranks. Indeed every element $C(\eta)$ can be represented as $C(\eta)=C\left(\eta_{n}\right) \cap C\left(\eta_{n+1}\right) \cap \ldots$ where $C\left(\eta_{n}\right)$ is a non-adjacent element of $\eta_{n}$ and $r_{+}\left(C\left(\eta_{n}\right)\right)=r_{+}\left(C\left(\eta_{n+1}\right)\right)=\ldots, r_{-}\left(C\left(\eta_{n}\right)\right)=r_{-}\left(C\left(\eta_{n+1}\right)\right)=\ldots$ and do not depend on $n$. Therefore we can put $r_{+}(C(\eta))=r_{+}\left(C\left(\eta_{n}\right)\right), r_{-}(C(\eta))=r_{-}\left(C\left(\eta_{n}\right)\right)$. Let $M_{k,+}\left(M_{k,-}\right)$ be the set of $C(\eta)$ for which $r_{+}(C(\eta))=k\left(r_{-}(C(\eta))=k\right)$.

Lemma 5.4. For sufficiently large $k$ and some $\alpha, 0<\alpha<1$,

$$
v\left(M_{k,+}\right) \leqq \alpha^{k}, \quad v\left(M_{k,-}\right) \leqq \alpha^{k} .
$$

Proof. Let $\Pi_{n}$ be the union of all elements of $\eta_{n}$ adjacent to $\bigcup_{-m \leqq k \leqq m n} S_{k}, v\left(\Pi_{n}\right)=\beta_{n}$. It is obvious from the construction that $v\left(M_{k,+}\right) \leqq v\left(\Pi_{k}\right)$. From the contractive properties of $T$ it follows that

$$
v\left(\bigcup_{0 \leqq s \leqq k-1} T_{1}^{s} \mathscr{D}_{k}^{(m)}\right) \leqq \Lambda_{\min }^{-m} \beta_{k-1} .
$$


In view of the measure preserving property of $T_{1}$ we have

$$
T_{1}^{k} \mathscr{D}_{k}^{(m)} \cong \Lambda_{\min }^{-m} \beta_{k-1}
$$

From the construction it follows that $\Pi_{k}=\bigcup_{0 \leqq s \leqq k} T_{1}^{s} \mathscr{D}_{k}^{(m)}$.

So we obtain

$$
v\left(M_{k,+}\right) \leqq 2 \Lambda_{\min }^{-m} \beta_{k-1}
$$

This inequality can likewise be proved for the set $M_{k,-}$. Q.E.D.

We denote by $k_{0}$ a minimal natural number for which $v\left(M_{k_{0},+}\right)>0$, $v\left(M_{k_{0},-}\right)>0$.

Lemma 5.5. $\eta$ is a countable partition.

Proof. It is sufficient to prove the assertion of lemma for partition $\eta^{+}=\bigvee_{n} \eta_{n}^{+}$. Let us put $\theta_{n}=v\left(\mathscr{D}_{n}^{(m)}\right), v_{n}=v\left(\bigcup_{0 \leqq k \leqq n} T_{1}^{k} \mathscr{D}_{n}^{(m)}\right)$. It is easy to see that $\sum_{n} v_{n}<\infty$. We shall give an abstract version of the assertion which we need.

Suppose that we have a sequence of finite partitions $\xi_{1} \leqq \xi_{2} \leqq \ldots \leqq \xi_{n} \leqq \ldots$ of a measure space $\left(M_{1}, \mathfrak{H}, v\right)$ and a measure-preserving transformation $T_{1}$ with the following properties: for each $n$ there exists a set $\mathscr{D}_{n}$ of elements of $\xi_{n}$ such that if $\mathscr{F}_{n}=\bigcup_{0 \leqq k \leqq n} T_{1}^{k} \mathscr{D}_{n}$ then $\mathscr{F}_{n}$ consists of elements of $\xi_{n+1}$ and $\xi_{n+1} \mid\left(M_{1}-\mathscr{F}_{n}\right)$ $=\xi_{n} \mid\left(M_{1}-\mathscr{F}_{n}\right)$. We shall show that $\bigvee_{n} \xi_{n}$ is a countable partition provided $\sum v\left(\mathscr{F}_{n}\right)<\infty$.

In order to show the last statement we put $\mathscr{E}_{n}=\bigcup_{k \geqq n} \mathscr{F}_{k}$ and $M_{1 n}=M_{1}-\mathscr{E}_{n}$. Then $M_{1}-\mathscr{E}_{n}$ consists obviously of elements of the partition $\bigvee_{n} \xi_{n}, \xi_{n_{1}} \mid M_{1 n}$ $=\xi_{n} \mid M_{1 n}$ for all $n_{1} \geqq n$ and $\underset{n_{1} \geqq n}{\bigvee} \xi_{n_{1}}\left|M_{1 n}=\xi_{n}\right| M_{1 n}$. Thus $\bigvee_{n_{1}} \xi_{n_{1}} \mid M_{1 n}$ for all $n_{1} \geqq n$ is equal to $\xi_{n} \mid M_{1 n}$. But $\xi_{n} \mid M_{1 n}$ is a finite partition. From our conditions it follows that $v\left(\mathscr{E}_{n}\right) \rightarrow 0$.

The statement of the lemma for $\left\{\eta_{n}^{+}\right\}$is a particular case of this general assertion and therefore $\eta^{+}=\bigvee_{n} \eta_{n}^{+}$is a countable partition. In the same way $\eta^{-}=\bigvee_{n} \eta_{n}^{-}$is also a countable partition. Therefore $\eta=\eta^{+} \vee \eta^{-}$is also a countable partition. Q.E.D.

Lemma 5.6. $\eta$ is a Markov partition.

Proof. We must show only that every element of $\eta$ is a parallelogram. Let $x \in C(\eta)$, $r_{+}=r_{+}(C(\eta)), r_{-}=r_{-}(C(\eta)), C(\eta)=C\left(\eta^{+}\right) \cap C\left(\eta^{-}\right)$. It follows from the construction that $C\left(\eta^{+}\right) C C\left(\eta_{n}^{+}\right), r_{+}\left(C\left(\eta_{n}^{+}\right)\right)=r_{+}$for all sufficiently large $n$. Let $k$ be equal to the minimum of all such $n$. It is easy to see that $k \geqq\left[\frac{1}{2} r_{+}\right]$. From Lemma $5.2 C\left(\eta_{k}^{+}\right)$is a quadrilateral. Therefore it is sufficient to show that if $\gamma^{(u)}(x)$ is a maximal smooth 
unstable transversal curve for $x$ then $\gamma^{(u)}(x)$ intersects both components of the boundary $\Gamma^{(s)}\left(C\left(\eta_{k}^{+}\right)\right)$.

If it were wrong then $\gamma^{(u)}(x)$ would intersect $\Gamma^{(u)}\left(C\left(\eta_{k}^{+}\right)\right)$or $\Gamma^{(u)}\left(C\left(\eta_{s}^{+}\right)\right)$for some $s>k$ and $x \in C\left(\eta_{s}^{+}\right) C C\left(\eta_{k}^{+}\right)$. We have seen above during the proof of Lemma 5.2 that $C\left(\eta_{k}^{+}\right)-C\left(\eta_{s}^{+}\right)$consisted of a finite number of strips whose stable boundaries belonged to $\Gamma^{(s)}\left(C\left(\eta_{k}^{+}\right)\right)$. Let $\gamma_{1}^{(u)}(x)$ be the connected component of $\gamma^{(u)}(x), x \in \gamma_{1}^{(u)}(x)$ and at least one of the end-points of $\gamma_{1}^{(u)}(x)$ belongs to $\Gamma^{(u)}\left(C\left(\eta_{s}^{+}\right)\right)$. The curve $\gamma_{1}^{(u)}(x)$ cut the connected component of $C\left(\eta_{s}^{+}\right)$containing $x$ into two parts which we denote by $A$ and $B$. We remark now that $C\left(\eta_{s}^{+}\right)-C(\eta)$ is a union of an infinite number of similar strips as above and this union is everywhere dense in $C\left(\eta_{s}^{+}\right)$. It means that infinitely many strips will intersect $\gamma_{1}^{(u)}(x)$ and therefore $\gamma_{1}^{(u)}(x)$ will intersect at infinitely many points the set $\bigcup_{s m+1 \leqq k<\infty} S_{k}$. But this is impossible because the inner part of $\gamma_{1}^{(u)}(x)$ cannot contain the points of $\bigcup_{s m+1 \leqq k<\infty} S_{k}$ because every intersection makes a break of the derivative of $\gamma_{1}^{(u)}(x)$ which contradicts the smoothness of $\gamma_{1}^{(u)}(x)$.

The same arguments show that $\gamma^{(s)}(x)$ intersects both components of $\Gamma^{(u)}\left(C\left(\eta_{k}^{-}\right)\right)$ and therefore $C(\eta)=C_{1}\left(\eta^{+}\right) \cap C_{2}\left(\eta^{-}\right)$are parallelograms. Q.E.D.

\section{Symbolic Dynamics and Other Properties of the Partition $\eta$}

Let $\eta$ be the Markov partition constructed in the preceeding section. We label the elements of $C(\eta)$ by natural numbers, i.e. $C_{i}(\eta)=C_{i}, i \geqq 1$. The \pm -ranks become the functions of $i$ which we denote by $r_{+}(i), r_{-}(i)$. We can choose the labelling in such a way that $r_{+}\left(i_{1}\right) \geqq r_{+}\left(i_{2}\right)$ if $i_{1} \geqq i_{2} . \xi^{(s)}, \xi^{(u)}$ are the measurable partitions of $M_{1}$ whose elements are maximal regular 1.s.t.f. and 1.u.t.f. $\gamma^{(s)}(x), \quad \gamma^{(u)}(x), \quad \eta^{(s)}=\xi^{(s)} \vee \eta$, $\eta^{(u)}=\xi^{(u)} \vee \eta$. A parallelogram $C$ is $u$-embedded in a parallelogram $\mathscr{D}$ if $C \leqq \mathscr{D}$ and for every $x \in C$ we have $\gamma_{C}^{(u)}(x)=\gamma_{\mathscr{D}}^{(u)}(x)$. In an analogous way one can introduce the notion of $s$-embedding.

The content of this section is similar in many respects to the corresponding parts of $[2,3]$. Therefore we shall omit some details in the proofs which are the same as in $[2,3]$.

If $\varepsilon$ in the construction of $\eta_{0}$ is small enough then an infinite product $\bigcap_{-\infty}^{\infty} T_{1}^{s} C_{i_{s}}(\eta)$ cannot consists of more than one point. If it consists of one point then all intersections $C_{i_{s}}(\eta) \cap T_{1} C_{i_{s+1}}(\eta) \neq \emptyset,-\infty<s<\infty$. The inverse assertion is also true: suppose that for a sequence of elements $C_{i_{s}}(\eta)$ all $v\left(C_{i_{s}}(\eta) \cap T_{1} C_{i_{s+1}}(\eta)\right)>0$; then the product $\bigcap_{-\infty}^{\infty} T_{1}^{s} C_{i_{s}}(\eta) \neq \emptyset$. The usual proof (see $[2,3]$ ) is based upon the canonical isomorphism between different $\gamma_{1}^{(s)}, \gamma_{2}^{(s)} \subset C_{i}(\eta)$ and can be applied in our case too. Therefore let us introduce the matrix of intersections $\Pi=\left\|\pi_{i j}\right\|$ where $\pi_{i j}=1$ if $v\left(C_{i} \cap T_{1} C_{j}\right)>0$ and 0 otherwise. We construct the space $\Omega_{\Pi}$ of sequences $\omega=\left\{\ldots, \omega_{-n}, \ldots, \omega_{0}, \ldots, \omega_{n}, \ldots\right\}, \omega_{i}=1,2, \ldots$ and $\pi_{\omega_{n} \omega_{n+1}}=1,-\infty<n<\infty$. If we introduce discrete topology in the space of natural numbers then $\Omega_{\Pi I}$ will become a 
topological space with the topology of the direct product of topological spaces. The space $\Omega_{\Pi}$ is invariant under the shift $T_{\Pi}$ in $\Omega_{\Pi}$.

As in $[2,3]$ we define the mappings $\varphi: \Omega_{\Pi} \rightarrow M_{1}$ putting $\varphi(\omega)=\bigcap_{-\infty}^{\infty} T_{1}^{n} C_{\omega_{n}}(\eta)$. It follows from the Markov properties of $\eta$ that $\varphi$ is defined everywhere on $\Omega_{\Pi}$.

Lemma 6.1. The mapping $\varphi$ is one-to-one mapping of $\Omega_{\Pi}$ onto its image. The image $\varphi\left(\Omega_{\Pi}\right)$ is a subset of $M_{1}$ of full measure. The inverse mapping $\varphi^{-1}$ is continuous.

Proof of Lemma 6.1 is the same as the proof of the Theorem 3.1 in [2].

Using $\varphi$ one can introduce the induced measure $v_{\Omega}$ on $\Omega_{\Pi}$ via the formula $v_{\Omega}(C)$ $=v(\varphi(C))$. From Lemma 6.1 it follows that $\varphi$ is an isomorphism of measure spaces $\left(M_{1}, \mathfrak{A}, v\right)$ and $\left(\Omega_{\Pi}, \mathfrak{X}_{\Pi}, v_{\Omega}\right)$ where $\mathfrak{I}_{\Pi}$ is the completion of the Borel $\sigma$-algebra of $\Omega_{\Pi}$ using $v_{\Omega}$. It follows from the relation $\varphi T_{1}=T_{\Pi} \varphi$ that $T_{\Pi}$ preserves the measure $v_{\Omega}$.

The triple $\left(\Omega_{\Pi}, \mathfrak{U}_{\Pi}, v_{\Omega}\right)$ is called a symbolic representation of the initial $\left(M_{1}, \mathfrak{A}, v\right)$. The rest of this section is devoted to the analysis of properties of the measure space $\left(\Omega_{\Pi}, \mathfrak{A}_{\Pi}, v_{\Omega}\right)$. It is based upon two facts.

1. Let us introduce measurable partitions $\zeta^{+}, \zeta^{-}$of $\Omega_{I I}$ where an element $C_{\zeta^{+}}\left(C_{\zeta^{-}}\right)$is defined by a semi-infinite subsequence $\omega_{0}, \omega_{1}, \ldots, \omega_{n}, \ldots$ $\left(\ldots \omega_{-n}, \ldots, \omega_{-1}, \omega_{0}\right)$ and consists of all $\omega$ which have this subsequence on corresponding places. An element of $\zeta^{+}\left(\zeta^{-}\right)$containing $\omega \in \Omega_{\Pi}$ will be denoted by $C_{\zeta^{+}}(\omega)\left(C_{\zeta^{-}}(\omega)\right)$. If $\omega \in C_{\zeta^{+}}, C_{\zeta^{-}}$and $\varphi(\omega)=x \in M_{1}$ then $\varphi\left(C_{\zeta^{+}}(\omega)\right)=C_{\eta^{(u)}}(x)$, $\varphi\left(C_{\zeta-}(\omega)\right)=C_{\eta^{(s)}}(x)$.

2. Let $\omega=\left\{\omega_{i}\right\},-\infty<i<\infty, r_{+}\left(\omega_{i}\right)=r_{+}\left(C_{\omega_{1}}\right)$.

If $r_{+}\left(\omega_{i}\right)=k>k_{0}, r_{-}\left(\omega_{i}\right)=\bar{k}>k_{0}$ then from Lemma $5.3 \quad r_{+}\left(\omega_{i-1}\right)=k-1$, $r_{-}\left(\omega_{i+1}\right)=\bar{k}-1$.

Lemma 6.2. There exists a constant $\alpha_{1}, 0<\alpha_{1}<1$, such that for all sufficiently large $r$

$$
v_{\Omega}\left(\omega: r_{+}\left(\omega_{0}\right) \geqq r\right) \leqq \alpha_{1}^{r}, \quad v_{\Omega}\left(\omega: r_{-}\left(\omega_{0}\right) \geqq r\right) \leqq \alpha_{1}^{r} .
$$

The statement of the lemma is a reformulation of Lemma 5.4.

Let $A_{n_{1} n_{2}}$ be the set of all $C_{i}=C_{i}(\eta)$ such that $r_{+}(i) \leqq n_{1}, r_{-}(i) \leqq n_{2}$.

Lemma 6.3. There exists a constant $k=k\left(n_{1}, n_{2}\right)$ with the following property: if $C_{\eta^{(u)}}$ $\in C(\eta) \subset A_{n_{1} n_{2}}$ then $v\left(C_{i} \mid T_{1}^{k} C_{\eta^{(u)}}\right)>0$ for every $C_{i} \subset A_{n_{1} n_{2}}$. The same statement is true for $\eta^{(s)}$.

The proof follows easily from the fact that $\eta^{(u)}, \eta^{(s)}$ are $K$-partitions for $T_{1}$ $[1,6]$. The next lemma estimates the growth of $k\left(n_{1}, n_{2}\right)$.

Lemma 6.4. There exists a constant $k_{0}$ such that

$$
k\left(n_{1}, n_{2}\right)=2 k_{0}+n_{1}+n_{2} .
$$


Proof. Let $r_{+}=\min _{i} r_{+}(i), r_{-}=\min _{i} r_{-}(i), k_{0}=k\left(r_{+}, r_{-}\right)$. If $C_{i}(\eta)$ is an element of $\eta$ then it follows from the construction of $\eta$ that for some $s$

$$
r_{+}(i)-r_{+} \leqq s \leqq r_{+}(i), \quad T_{1}^{s} C_{i}(\eta) \subset C_{j}(\eta) \subset A_{r_{+}, r_{-}} .
$$

The parallelogram $T_{1}^{s} C_{i}(\eta)$ is $u$-embedded in $C_{j}(\eta)$ due to the Markov properties of $\eta$. For the same reason $T_{1}^{s+k_{0}} C_{i}(\eta) \cap C_{k}(\eta)$ is a parallelogram of positive measure $u$-embedded in $C_{k}(\eta)$ for every $C_{k}(\eta) \subseteq A_{r_{+}, r_{-}}$. In the same way for an arbitrary $C_{i_{1}}(\eta) \subset A_{n_{1}, n_{2}}$ one can find $s_{1}, r_{-}\left(i_{1}\right)-r_{-} \leqq s_{1} \leqq r_{-}\left(i_{1}\right)$ such that $T_{1}^{-s_{1}} C_{i_{1}}(\eta) \subseteq C_{j_{1}}(\eta) \cong A_{r_{+}, r_{-}}$. The parallelogram $T_{1}^{-s_{1}} C_{i_{1}}(\eta)$ is $s$-embedded in $C_{j_{1}}(\eta)$ due to the Markov properties of $\eta$. Therefore $v\left(T_{1}^{-s_{1}} C_{i_{1}}(\eta)\right.$ $\left.\cap T_{1}^{s+k_{0}} C_{i}(\eta)\right)>0$, i.e. $v\left(C_{i_{1}}(\eta) \cap T_{1}^{s+s_{1}+k_{0}} C_{i}(\eta)\right)>0$. But it follows from the fact that $C_{i_{1}}(\eta), C(\eta)$ are parallelograms that $v\left(C_{i_{1}}(\eta) \mid T_{1}^{s_{1}+k_{0}} C_{\eta^{(u)}}\right)>0$. Q.E.D.

Our next task is to investigate one-sided conditional probabilities $v_{\Omega}\left(\omega_{0} \mid \omega_{-1}, \ldots, \omega_{-n}, \ldots\right)$ existing with $v_{\Omega}$-probability one. In case of Markov chains of finite memory this probability depends only on a finite number of coordinates. We shall show that in our case these conditional probabilities can be in a sense very well approximated by probabilities with finite memory. Taking constants $\alpha_{20}, \alpha_{21}, \alpha_{22}, 0<\alpha_{20}, \alpha_{21}, \alpha_{22}<1$ we introduce the sets:

$$
\begin{gathered}
U_{m}=\left\{x: \operatorname{dist}\left(x, S_{0}\right)<\alpha_{20}^{m}\right\}, \\
V_{n}=\left\{x: T^{k} x \notin U_{m}, m=\left[n^{\alpha_{21}}\right],|k| \leqq n \text { and } T^{i} x \in U_{i} \text { for }|i|>n\right\}, \\
W_{n}=\left\{x: v\left(V_{n} \mid C_{\eta^{(s)}}(x)\right)>1-\alpha_{22}^{n^{1 / 2}}\right\} .
\end{gathered}
$$

It is clear that $W_{n}$ consists of elements of $\eta^{(s)}$.

Lemma 6.5. Let $C_{\zeta^{-}}^{\prime}, C_{\zeta^{-}}^{\prime \prime} \cdot$ correspond to $\omega_{0}, \omega_{-1}, \ldots, \omega_{-n+1}, \omega_{-n}^{\prime}, \omega_{-n-1}^{\prime}, \ldots$; $\omega_{0}, \omega_{-1}, \ldots, \omega_{-n+1}, \omega_{-n}^{\prime \prime}, \omega_{-n-1}^{\prime \prime}, \ldots$ and $C_{\zeta^{-}}^{\prime}, C_{\zeta^{-}}^{\prime \prime} \in \varphi^{-1}\left(W_{n}\right)$. Then one can chose $\alpha_{20}, \alpha_{21}, \alpha_{22}$ and $\alpha_{2}, 0<\alpha_{2}<1$, in such a way that

$$
\sum_{\omega_{1}}\left|v\left(\omega_{1} \mid C_{\zeta^{-}}^{\prime}\right)-v\left(\omega_{1} \mid C_{\zeta^{-}}^{\prime \prime}\right)\right|<\alpha_{2}^{n^{1 / 2}}
$$

The proof of the lemma is based on properties of the canonical isomorphism of different $C_{\eta^{(s)}}^{\prime}, C_{\eta^{(s)}}^{\prime \prime}$ lying in the same element of the Markov partition $\eta$. The canonical isomorphism (see $[2,5]$ ) transforms the conditional measure on $C_{\eta^{(s)}}^{\prime \prime}$ induced by $v$ into a measure which is absolutely continuous with respect to the analogous conditional measure on $C_{\eta^{(s)}}^{\prime}$. For $C_{\eta^{(s)}}^{\prime}, C_{\eta^{(s)}}^{\prime \prime} \in W_{n}$ the corresponding Jacobian differs from one by a number whose modulo is no more than $\alpha_{3}^{n}$ for some $\alpha_{3}, 0<\alpha_{3}<1$, for points belonging to $V_{n}$. Indeed, it can easily be estimated on the basis of the formula for the Jacobian (see [5]). The last statement is equivalent to the assertion of Lemma 6.5. In terminology of statistical mechanics Lemma 6.5 shows that the potential $-\ln v_{\Omega}\left(\omega_{1} \mid \omega_{0}, \omega_{-1}, \ldots\right)$ for which $v_{\Omega}$ can be considered as a limit Gibbs state (see [8]) is of a short-range character. Further we assume that $\alpha_{20}, \alpha_{21}, \alpha_{22}, \alpha_{2}$ are chosen in such a way that Lemma 6.5 is valid. 
The last property which we shall consider is an analogy of the so-called Doeblin's condition in the theory of usual Markov chains. Let us introduce conditional probabilities

$$
\begin{aligned}
\pi_{1}\left(\omega_{3 n+1}, \ldots, \omega_{4 n}\right) & =v_{\Omega}\left(\omega_{3 n+1}, \ldots, \omega_{4 n} \mid \omega_{-n+1}^{\prime}, \ldots, \omega_{0}^{\prime}\right), \pi_{2}\left(\omega_{3 n+1}, \ldots, \omega_{4 n}\right) \\
& =v_{\Omega}\left(\omega_{3 n+1}, \ldots, \omega_{4 n} \mid \omega_{-n+1}^{\prime \prime}, \ldots, \omega_{0}^{\prime \prime}\right) .
\end{aligned}
$$

We have the probability distributions $\pi_{1}, \pi_{2}$ on the space of words $\omega_{3 n+1}, \ldots, \omega_{4 n}$ under different conditions $\omega_{-n+1}^{\prime}, \ldots, \omega_{0}^{\prime}$ and $\omega_{-n+1}^{\prime \prime}, \ldots, \omega_{0}^{\prime \prime}$.

Lemma 6.6. Suppose that $r_{ \pm}\left(\omega_{i}^{\prime}\right) \leqq n, r_{ \pm}\left(\omega_{i}^{\prime \prime}\right) \leqq n, 1 \leqq i \leqq n$. Then there exists a constant $\alpha_{4}, 0<\alpha_{4}<1$, such that for all large enough $n$

$$
\operatorname{Var}\left(\pi_{1}, \pi_{2}\right)=\frac{1}{2} \sum_{\omega_{3 n+1}, \ldots, \omega_{4 n}}\left|\pi_{1}\left(\omega_{3 n+1}, \ldots, \omega_{4 n}\right)-\pi_{2}\left(\omega_{3 n+1}, \ldots, \omega_{4 n}\right)\right|<\alpha_{4} .
$$

Proof consists of three steps.

1. It follows from the construction of $\eta$ that there exists a finite collection of elements of $\eta A=\left(C_{1}(\eta), \ldots, C_{m}(\eta)\right)$ such that for every $C(\eta) \notin \bigcup_{i=1}^{m} C_{i}(\eta) T C(\eta)$ is another element of $\eta$.

Let us consider two $C_{\eta^{(s)}}^{\prime}, C_{\eta^{(s)}}^{\prime \prime} \subset C_{i}(\eta)$ for some $i, 1 \leqq i \leqq m$. They are canonically isomorphic because $C_{i}(\eta)$ is a parallelogram. The induced conditional distributions are equivalent and the corresponding density depends on expansion coefficients along the semi-trajectories (see [5] where one can find an exact expression for the density in case of Anosov systems; the same expression is valid in our case too). It means that for every $d, 1<d<\infty$, one can find a parallelogram $\mathscr{D}_{i} \subset C_{i}(\eta), 1 \leqq i \leqq m$, which is $u$-embedded in $C_{i}(\eta)$ such that $v\left(\mathscr{D}_{i}\right) \geqq\left(1-d^{-1}\right) v\left(C_{i}(\eta)\right)$ and the restriction of the density to $\mathscr{D}_{i} \cap C_{\eta^{(s)}}^{\prime}$ is contained between $d^{-1}$ and $d$ uniformly over $C_{\eta^{(s)}}^{\prime}, C_{\eta^{(s)}}^{\prime \prime}$. Thus $\mu\left(\mathscr{D}_{i} \mid C_{\left.\eta^{(s)}\right)} \geqq \gamma_{1}>0\right.$ for all $C_{\eta^{(s)}} \subset C_{i}(\eta)$ where $\gamma_{1}=\gamma_{1}(d)$ depend on $d$. Using the language of symbolic dynamics one can say that

$$
d^{-1} \leqq \frac{v_{\Omega}\left(\omega_{k}, \omega_{k+1}, \ldots, \omega_{l} \mid C_{\zeta-}^{\prime} \cap \varphi^{-1}\left(\mathscr{D}_{i}\right)\right)}{v_{\Omega}\left(\omega_{k}, \omega_{k+1}, \ldots, \omega_{l} \mid C_{\zeta-}^{\prime \prime} \cap \varphi^{-1}\left(\mathscr{D}_{i}\right)\right)} \leqq d
$$

for arbitrary $k, l, k<l$. Here $C_{\zeta^{-}}^{\prime}=\varphi^{-1}\left(C_{\eta^{(s)}}^{\prime}\right), C_{\zeta^{-}}^{\prime \prime}=\varphi^{-1}\left(C_{\left.\eta^{(s)}\right)}^{\prime \prime}\right.$. It is also obvious that one can take as a condition $\varphi^{-1}\left(\mathscr{D}_{i}\right)$ instead of $C_{\zeta^{-}}^{\prime} \cap \varphi^{-1}\left(\mathscr{D}_{i}\right)$ and the same inequalities will be valid.

2. Let $C_{\eta^{(s)}} C C(\eta)$ and $r_{ \pm}(C(\eta)) \leqq n$. We shall show that one can find $l_{0}<3 n$ and $\alpha_{5}, 0<\alpha_{5}<1$, not depending on $n$ such that for all $l>l_{0}$

$$
v\left(T_{1}^{l} C_{i}(\eta) \mid C_{\eta^{(s)}}\right)=v\left(C_{i}(\eta) \mid T_{1}^{-l} C_{\eta^{(s)}}\right) \geqq \alpha_{5}, \quad 1 \leqq i \leqq m .
$$

Indeed from Lemma 6.4 one can find $l_{1} \leqq 2 n+k_{0}$, for which $T_{1}^{-l_{1}} C_{\eta^{(s)}}=C_{\eta^{(s)}}^{\prime} \subset C_{i}(\eta)$ for some $i, 1 \leqq i \leqq m$. Let be $l \geqq l_{0}>l_{1}$ and $l_{0}-l_{1}$ be large enough. We have

$$
\begin{gathered}
v\left(T_{1}^{l} C_{j}(\eta) \mid C_{\eta^{(s)}}\right)=v\left(T_{1}^{l-l_{1}} C_{j}(\eta) \mid T_{1}^{-l_{1}} C_{\eta^{(s)}}\right) \geqq v\left(T_{1}^{l-l_{1}} C_{j}(\eta) \cap \mathscr{D}_{i} \mid C_{\eta^{(s)}}^{\prime}\right) \\
\geqq d^{-2} v\left(T_{1}^{l-l_{1}} C_{j}(\eta) \mid \mathscr{D}_{i}\right) .
\end{gathered}
$$


Because $T_{1}$ is mixing $v\left(T_{1}^{l-l_{1}} C_{j}(\eta) \mid \mathscr{D}_{i}\right) \geqq \frac{1}{2} v\left(C_{j}(\eta)\right)$. Also for all $l \geqq l_{0}$

$$
v\left(T_{1}^{l-l_{1}} C_{j}(\eta) \mid T^{-l_{1}} C_{\eta^{(s)}}\right) \geqq d^{-2} v\left(T_{1}^{l-l_{1}} C_{j}(\eta) \mid \mathscr{D}_{i}\right) \geqq \frac{d^{-2}}{2} \min _{1 \leqq j \leqq m} v\left(C_{j}(\eta)\right) .
$$

Putting $\alpha_{5}=\frac{1}{2} d^{-2} \min _{1 \leqq j \leqq m} v\left(C_{j}(\eta)\right)$ we get the desired inequality.

3. Let $B$ be a set of the form $\bigcap_{i=3 n+1}^{4 n} T_{1}^{i} C_{\omega_{i}}(\eta)$. We have

$$
V=\operatorname{Var}\left(\pi_{1}, \pi_{2}\right)=\sum_{B}^{+}\left(v\left(\left.B\right|_{i=0} ^{n-1} T_{1}^{-i} C_{\omega_{i}}(\eta)\right)-v\left(B \mid \bigcap_{i=0}^{n-1} T_{1}^{-i} C_{\omega^{\prime \prime}}(\eta)\right)\right),
$$

where $\sum^{+}$here and further means that the summation is taken over positive terms. We can write

$$
V=\sum_{B}^{+}\left[\int_{C_{\eta^{(s)}}^{\prime} C_{i=0}^{n-1} \bigcap_{l=0}^{1} T_{1}^{-1} C_{\omega_{i}^{\prime}}^{(\eta)}} v\left(B \mid C_{\eta^{(s)}}^{\prime}\right) d v^{\prime}-\int_{C_{\eta^{(s)}}^{\prime \prime} \subset \bigcap_{i=0}^{n-1} T_{1}^{-i} C_{\omega_{i}^{\prime \prime}(\eta)}} v\left(B \mid C_{\eta^{(s)}}^{\prime \prime}\right) d v^{\prime \prime}\right]
$$
where $d v^{\prime}, d v^{\prime \prime}$ are normed measures on $C_{\eta^{(s)}}^{\prime} \subset \bigcap_{i=0}^{n-1} T_{1}^{-i} C_{\omega_{t}^{\prime}}(\eta), C_{\eta^{(s)}}^{\prime \prime} \subset \bigcap_{i=0}^{n-1} T_{1}^{-i} C_{\omega_{i}^{\prime \prime}}(\eta)$
respectively. Further

$$
\begin{aligned}
& V \leqq \sum_{B}\left[\int_{\bigcap_{i=0}^{n-1} T_{1}^{-i} C_{\omega_{i}^{\prime}}(\eta)} v\left(B \mid\left(M_{1}-\bigcup_{i=1}^{m} T_{1}^{3 n} \mathscr{D}_{i}\right) \cap C_{\eta^{(s)}}^{\prime}\right) \cdot v\left(\left(M_{1}-\bigcup_{i=1}^{m} T_{1}^{3 n} \mathscr{D}_{i}\right) \mid C_{\eta^{(s)}}^{\prime}\right) d v^{\prime}\right.
\end{aligned}
$$

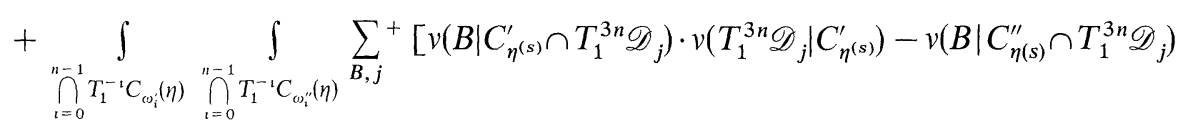

$$
\begin{aligned}
& \left.\cdot v\left(T_{1}^{3 n} \mathscr{D}_{j} \mid C_{\eta^{\prime}(s)}^{\prime \prime}\right)\right] d v^{\prime} d v^{\prime \prime}=v\left(\left.\left(M_{1}-\bigcup_{i=1}^{m} T_{1}^{3 n} \mathscr{D}_{i}\right)\right|_{i=0} ^{n-1} \bigcap_{1}^{-i} C_{\omega^{\prime}}(\eta)\right) \\
& +\int_{\bigcap_{i=0}^{n-1} T_{1}^{-i} C_{\omega_{i}^{\prime}(\eta)}} \int_{\bigcap_{i=0}^{n-1} T_{1}^{-\imath} C_{\omega_{i}^{\prime}}(\eta)} \sum_{B, j}^{+} v\left(B \mid C_{\eta^{(s)}}^{\prime} \cap T_{1}^{3 n} \mathscr{D}_{j}\right) \cdot v\left(T_{1}^{3 n} \mathscr{D}_{j} \mid C_{\eta^{(s)}}^{\prime}\right) \\
& \left.\cdot\left[1-\frac{v\left(B \mid C_{\eta^{(s)}}^{\prime \prime} \cap T_{1}^{3 n} \mathscr{D}_{j}\right)}{v\left(B \mid C_{\eta^{(s)}}^{\prime} \cap T_{1}^{3 n} \mathscr{D}_{j}\right)} \frac{v\left(T_{1}^{3 n} \mathscr{D}_{j} \mid C_{\eta^{(s)}}^{\prime \prime}\right)}{v\left(T_{1}^{3 n} \mathscr{D}_{j} \mid C_{\eta^{(s)}}^{\prime}\right)}\right] d v^{\prime} d v^{\prime \prime}\right]
\end{aligned}
$$

We remark that $v\left(B \mid C_{\eta^{(s)}}^{\prime \prime} \cap T_{1}^{3 n} \mathscr{D}_{j}\right)=v\left(T_{1}^{-3 n} B \mid \mathscr{D}_{j} \cap T_{1}^{-3 n} C_{\eta^{(s)}}^{\prime \prime}\right)$. The set $C_{j}(\eta) \cap T_{1}^{-3 n} C_{\eta^{(s)}}^{\prime}$ is a finite union of some $\tilde{C}_{\eta^{(s)}}^{\prime}$. The set $\mathscr{D}_{j} \cap T_{1}^{-3 n} C_{\eta^{(s)}}^{\prime}$ is the same union of $\mathscr{D}_{j} \cap \mathcal{C}_{\eta^{(s)}}^{\prime}$. The same statement is valid for $C_{j}(\eta) \cap T_{1}^{-3 n} C_{\eta^{(s)}}^{\prime \prime}$. Now it follows from $1^{\circ}$ that

$$
v\left(B \mid C_{\eta^{(s)}}^{\prime \prime} \cap T_{1}^{3 n} \mathscr{D}_{j}\right) \geqq d^{-1} v\left(B \mid C_{\eta^{(s)}}^{\prime} \cap T_{1}^{3 n} \mathscr{D}_{j}\right) .
$$


Also from $1^{\circ}$ and $2^{\circ} v\left(T_{1}^{3 n} \mathscr{D}_{j} \mid C_{\eta^{(s)}}^{\prime}\right) / v\left(T_{1}^{3 n} \mathscr{D}_{j} \mid C_{\eta^{(s)}}^{\prime \prime}\right) \geqq \alpha_{5} \gamma_{1}=\gamma_{2}$. Therefore

$$
1-\frac{v\left(B \mid C_{\eta^{(s)}}^{\prime \prime} \cap T_{1}^{3 n} \mathscr{D}_{j}\right)}{v\left(B \mid C_{\eta^{(s)}}^{\prime} \cap T_{1}^{3 n} \mathscr{D}_{j}\right)} \cdot \frac{v\left(T_{1}^{3 n} \mathscr{D}_{j} \mid C_{\eta^{(s)}}^{\prime \prime}\right)}{v\left(T_{1}^{3 n} \mathscr{D}_{j} \mid C_{\eta^{(s)}}^{\prime}\right)} \leqq 1-\gamma_{2} d^{-1}
$$

and

$$
\begin{aligned}
V \leqq & \left(M_{1}-\bigcup_{i=1}^{m} T_{1}^{3 n} \mathscr{D}_{i} \mid \bigcap_{i=0}^{n-1} T_{1}^{-i} C_{\omega_{i}^{\prime}}(\eta)\right)+\left(1-\gamma_{2} d^{-1}\right)\left(1-v\left(M_{1}-\bigcup_{i=1}^{m} T_{1}^{3 n} \mathscr{D}_{i} \mid\right.\right. \\
& \left.\left.\cdot \bigcap_{i=0}^{n-1} T_{1}^{-i} C_{\omega_{i}^{\prime}}(\eta)\right)\right) \leqq 1-\gamma_{2} d^{-1}\left(1-v\left(M_{1}-\bigcup_{i=1}^{m} T_{1}^{3 n} \mathscr{D}_{i} \mid \bigcap_{i=0}^{n-1} T_{1}^{-i} C_{\omega_{i}^{\prime}}(\eta)\right)\right)
\end{aligned}
$$

But it follows easily from $2^{\circ}$ that $v\left(M_{1}-\left.\bigcup_{i=1}^{m} T_{1}^{3 n} \mathscr{D}_{i}\right|_{i=0} ^{n-1} T_{1}^{-i} C_{\omega_{i}^{\prime}}(\eta)\right) \leqq \gamma_{3}$ where $\gamma_{3} \rightarrow 0$ as $d \rightarrow \infty$. Therefore the last expression is uniformly less than 1. Q.E.D.

\section{Concluding Remarks}

$1^{\circ}$. We shall describe a more general case to which the whole construction can be applied. Suppose that we have an infinite configuration of convex non-overlapping scatterers on the plane $\mathbb{R}^{2}$ such that

1) the curvature of the boundary of each domain is continuous and bounded from above and from below by some positive constants;

2) the length of an arbitrary straight segment which does not intersect any of the scatterers is bounded from above and from below by some positive constants;

Let us consider the motion of a single particle between the scatterers with elastic reflections from the scatterers. We denote by $M_{1}^{(i)}$ the set of $x=(q, v)$ where $q$ is a point of the boundary of the $i$-th scatterer, $v$ is the velocity vector directed outside the scatterer. We put $M_{1}=\bigcup_{i} M_{1}^{(i)}$ and consider the transformation $T: M_{1} \rightarrow M_{1}$ generated by the motion of the particle. The transformation $T$ preserves the infinite measure $v$ whose restriction to $M_{1}^{(i)}$ takes the form $d v=d q d \varphi \cos \varphi$.

One can introduce the definition of Markov partition in the same way as in Definition 1. Our construction can be performed without any changes under assumptions 1), 2). This gives the existence of a Markov partition in this case too. The properties of this partition can be investigated in the same way as in Sect. 6 .

$2^{\circ}$. Let $\alpha$ be the transformation of $M_{1}$ which is induced by the involution in the phase space $M$ which is generated by changing $v$ into $-v$. It is easy to see that the set of discontinuity curves $\bigcup_{|k| \leqq m} S_{k}$ is invariant under $\alpha$. One can easily modify the construction of the Markov partition in such a way that it will be also invariant under $\alpha$. 


\section{References}

1. Sinai,Ya.G.: Russ. Math. Survey 25, 137-189 (1970)

2. Sinai, Ya.G.: Funct. Anal. Appl. 2, 64-89 (1968); 2, 70-80 (1968)

3. Bowen, R.: Equilibrium states and ergodic theory of Anosov diffeomorphisms. In: Lecture notes in mathematics, Vol. 470, p. 108. Berlin, Heidelberg, New York: Springer 1975

4. Ruelle, D.: Thermodynamic formalism, p. 180. New York: Addison-Wesley 1978

5. Anosov, D.V., Sinai, Ja.G. : Russ. Math. Survey 22, 103-167 (1967)

6. Gallavotti, G.: Lectures on billiards. In: Lecture notes in physics, Vol. 38, pp. 236-296. Berlin, Heidelberg, New York: Springer 1975

7. Keller, G.: Diplomarbeit, p. 203. Erlangen (1977)

8. Sinai,Ya.G.: Theory of phase transitions. Rigorous results, p. 160. Moscow: Nauka 1980

Communicated by A. Jaffe

Received April 29, 1980 